\title{
Short-Term Synaptic Depression Causes a Non-Monotonic Response to Correlated Stimuli
}

\author{
Jaime de la Rocha and Néstor Parga \\ Departamento de Física Teórica, Universidad Autónoma de Madrid, Canto-Blanco, 28049 Madrid, Spain
}

Unreliability is a ubiquitous feature of synaptic transmission in the brain. The information conveyed in the discharges of an ensemble of cells (e.g., in the spike count or in the timing of synchronous events) may not be faithfully transmitted to the postsynaptic cell because a large fraction of the spikes fail to elicit a synaptic response. In addition, short-term depression increases the failure rate with the presynaptic activity. We use a simple neuron model with stochastic depressing synapses to understand the transformations undergone by the spatiotemporal patterns of incoming spikes as these are first converted into synaptic current and afterward into the cell response. We analyze the mean and SD of the current produced by different stimuli with spatiotemporal correlations. We find that the mean, which carries information only about the spike count, rapidly saturates as the input rate increases. In contrast, the current deviation carries information about the correlations. If the afferent action potentials are uncorrelated, it saturates monotonically, whereas if they are correlated it increases, reaches a maximum, and then decreases to the value produced by the uncorrelated stimulus. This means that, at high input rates, depression erases from the synaptic current any trace of the spatiotemporal structure of the input. The non-monotonic behavior of the deviation can be inherited by the response rate provided that the mean current saturates below the current threshold setting the cell in the fluctuation-driven regimen. Afferent correlations therefore enable the modulation of the response beyond the saturation of the mean current.

Key words: synaptic integration; fluctuation-driven regimen; presynaptic spike correlations; synaptic short-term depression; vesicle depletion; neural coding

\section{Introduction}

Since the first studies on synaptic transmission in the neuromuscular junction, Katz and Miledi (1968) realized that transmitter release occurs stochastically. Additional studies found that central synapses are also very unreliable (Hessler et al., 1993; Rosenmund et al., 1993) and that the transmission probability undergoes temporary changes according to the recent activity (Magleby, 1987; Zucker and Regehr, 2002). This probability decreases under depletion of the releasable transmitter vesicles (Dobrunz and Stevens, 1997), giving rise to short-term synaptic depression (STD).

Several works have explored the theoretical implications of these findings on the transmission of information in neural circuits (Abbott et al., 1997; Lisman, 1997; Tsodyks and Markram, 1997; Matveev and Wang, 2000b). One of the most relevant consequences of STD is that the synapses saturate at presynaptic rates $(\nu)$ higher than a limiting rate, that is, the mean stationary afferent current $\left(\mu_{I}\right)$ does not depend on $\nu$. This saturation appears to be a major constraint on the range of $\nu$ within which neurons can

Received Feb. 16, 2005; revised July 19, 2005; accepted July 19, 2005.

This work was supported by a postdoctoral fellowship and Grant BFM 2003-06242 from the Spanish Ministry of Education and Science. We thank Alfonso Renart, Emilio Salinas, Angel Nevado, and Alex Reyes for critical reading of this manuscript.

Correspondence should be addressed to Jaime de la Rocha, Center for Neural Science, New York University, New York, NY 10003. E-mail:.jrocha@cns.nyu.edu.

DOI:10.1523/JNEUROSCI.0631-05.2005

Copyright $\odot 2005$ Society for Neuroscience $\quad 0270-6474 / 05 / 258416-16 \$ 15.00 / 0$ transmit information in the stationary regimen (Abbott et al., 1997; Tsodyks and Markram, 1997). However, this argument is based on the saturation of $\mu_{I}$ and neglects that the cell response is also determined by the current fluctuations $\left(\sigma_{I}\right)$. It may well be that $\sigma_{I}$ does not saturate as $\mu_{I}$ so that the response can be modulated beyond the limiting frequency.

The role of the current fluctuations has been attracting much attention recently (Chance et al., 2002; Kuhn et al., 2004; Moreno-Bote and Parga, 2005). They were proposed as a candidate mechanism to generate the large variability found in the activity of cortical cells (Softky and Koch, 1993), which would hypothetically operate in a fluctuation-driven regimen (FDR) (Gerstein and Mandelbrot, 1964; Shadlen and Newsome, 1994). There, the mean synaptic current generated by the network is not sufficient to drive the neurons toward threshold, and only the fluctuations may trigger discharges (van Vreeswijk and Sompolinsky, 1996). Depression transforms these fluctuations in a nontrivial manner; however, no studies have analyzed its impact on the neuron response.

Here, we use a computational model to study the role of the fluctuations on the stationary neuron response when the synapses are stochastic and show STD. We analyze the impact of STD on three factors: (1) the stochasticity of the transmission, (2) the redundant connectivity of the neurons (i.e., connections made of several contacts), and, most relevant, (3) the correlations between afferent spikes, analyzed using stimuli with different spatiotemporal structure. 

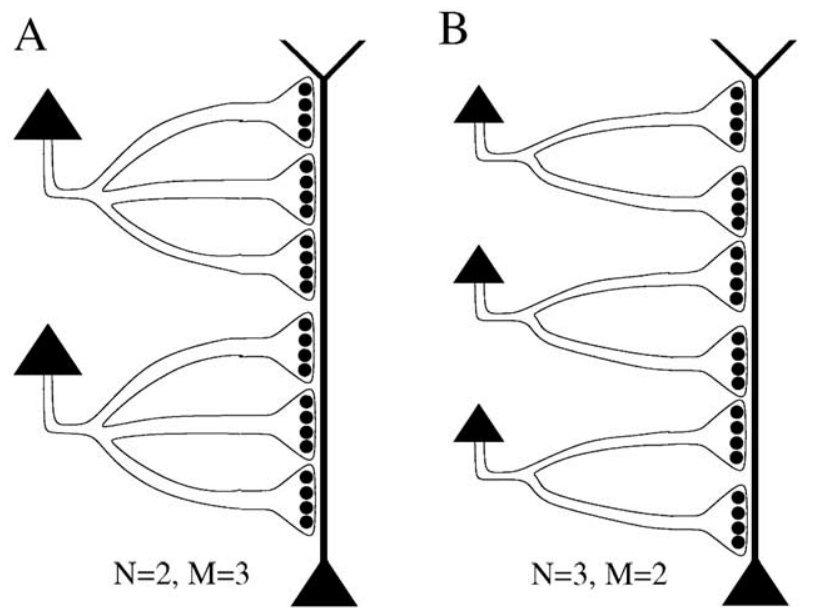

C

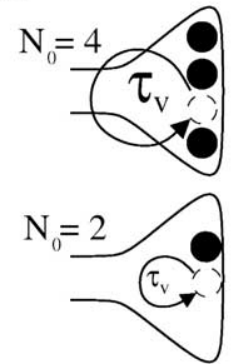

$\mathrm{D}$

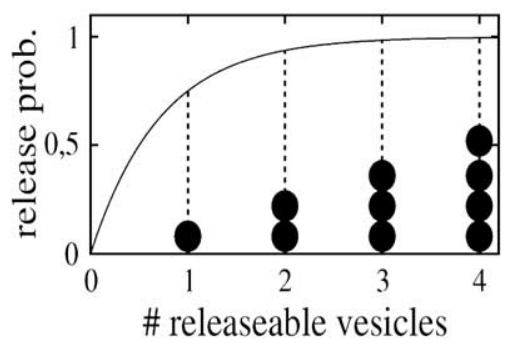

Figure 1. Illustrations of the synaptic connection characteristics in the stochastic model. $A$, $\boldsymbol{B}$, Two examples with different numbers of functional contacts per presynaptic cell ( $M=3$ and 2 , respectively). At each contact, an independent model of vesicle turnover is implemented with identical parameters. For the analysis performed in Figure 4, only the cases that, like these two examples, have the same total number of contacts $N \times M$ are compared. $C$, Recovery of a vacancy in the $\mathrm{RP}$ is stochastic and occurs independently at each vacancy. The mean recovery time $\tau_{v}$ is scaled with the size of the RP, so that cases with different $N_{0}$ can be compared fairly. $D$, Plot of the release probability (release prob.) versus the number of releasable vesicles (Eq. 1) with $U=0.75$ and $N_{0}=4$.

It will be shown that (1) STD may lead naturally to the FDR, because it automatically sets $\mu_{I}$ below the threshold current; (2) in this regimen, fluctuations can be modulated by the input rate even if the mean current has reached saturation; (3) at high rates, STD tends to eliminate the impact of the input correlations; and (4) as a result, correlations cause $\sigma_{I}$ to exhibit a non-monotonic behavior as a function of $\nu$, behavior that is inherited by the response rate.

Preliminary results have appeared in abstract form (Moreno et al., 2002a).

\section{Materials and Methods \\ The synapse model}

Because connected neurons rarely are connected by a single synapse, and synapses sometimes have more than one synaptic specialization (the particular area in which vesicle exocytosis takes place) (Walmsley et al., 1998), we will consider connections made of $M$ "functional contacts," the term used to refer to any synaptic specialization in which release takes place (Zador, 1998). Contacts sharing the same presynaptic cell will be referred to as "common" contacts. Figure $1, A$ and $B$, illustrates two examples in which each presynaptic cell establishes $M=3$ and $M=2$ common contacts, respectively. Cases with different values of $M$ will be compared at a fixed total number of contacts. This is done by varying the number of presynaptic neurons as in the examples shown in Figure 1, $A$ and $B$.

At each of these contacts, a simple stochastic model of vesicle turnover is implemented (Vere-Jones, 1966; Maass and Zador, 1999; Wang, 1999). The model assumes a vesicle "releasable pool" (RP) of size $N_{0}$, that is, it can hold up to $N_{0}$ vesicles. Vesicles within this pool are thought to be "docked" and will also be referred to as "readily releasable" vesicles. We assume the "univesicular release hypothesis," which states that, independently of the number of docked vesicles, an action potential (AP) can at most trigger the fusion of one vesicle per functional contact (Edwards et al., 1976). The release of a docked vesicle is modeled stochastically, that is, on arrival of a spike, a release is triggered with a certain probability. This probability is a function of the current number of releasable vesicles, $n$, as follows:

$$
p(n)=1-(1-U)^{n} \quad n=0,1, \ldots, N_{0}
$$

as proposed in a study of the hippocampal CA3-CA1 synapse (Dobrunz and Stevens, 1997; Dobrunz, 2002). This dependence on $n$ is illustrated in Figure $1 D$, where one can observe that the parameter $U$ represents the release probability when there is only one vesicle ready. The recovery of vesicles when the RP is not fully replenished is also modeled randomly: given that the number of vesicles ready to become docked is much larger than $N_{0}$ (De Camilli et al., 2001), the replenishment of a vacancy in the $\mathrm{RP}$ can be taken as the first event from a Poisson process with homogeneous mean recovery time $\tau_{v}$ (Fig. 1C). This modeling is compatible with the experimental observation that the recovery of the release probability from depletion can be fitted with an exponential (Dobrunz and Stevens, 1997) (data not shown). When comparing instances with RPs of different sizes, the recovery time $\tau_{v}$ will be normalized so that the ratio $\tau_{v} / N_{0}$ is the same in all of the examples. This normalization is sketched in the two examples shown in Figure $1 C$, in which the case with $N_{0}=4$ has a recovery time twice as large as that with $N_{0}=2$. This scaling makes the comparison between synapses with different $N_{0}$ values easier, because (as will be shown later) the current statistics in the limit of large $\nu$ depends only on the ratio $\tau_{v} / N_{0}$.

The average state of the synapses will be described by the probability that an afferent spike reaching a functional contact triggers the release of a vesicle. This "transmission probability" $\left(P_{t}\right)$ will be obtained by computing over a long stimulus the fraction of functional contacts that, being hit by an AP, elicit a synaptic response.

\section{Presynaptic activity: description of the stimuli}

We will consider four different types of presynaptic activity: (1) "uncorrelated" stimuli, (2) "synchronous" stimuli, (3) "autocorrelated" stimuli, and (4) "phase-locked periodic" stimuli.

Figure 2 shows a spike rastergram for each stimulus type, which serves to explain graphically the reason of their choice. Because spikes are independent, the raster obtained with the uncorrelated stimulus looks very homogeneous in comparison with the rest, because it lacks any kind of spatial or temporal structure (Fig. $2 A$ ). In contrast, the other three stimuli were chosen as simple examples with only spatial (Fig. $2 B$, synchronous), only temporal (Fig. $2 C$, autocorrelated), and both spatial and temporal structure (Fig. $2 D$, phase-locked). In each of these rasters, the corresponding structure is easily caught by the eye. Because of the presence of correlations, the global instantaneous activity of each stimulus (drawn below each raster), presents a different profile: despite its stochastic nature, the uncorrelated stimulus displays a much smaller temporal variability than the other three.

The stimuli are defined in terms of the "statistics" of the spike trains. Therefore, different trials do not reproduce the position of the spikes precisely, only the statistics of the presynaptic trains is the same. During a trial, each of the $N$ presynaptic neurons elicits a spike train composed of a sequence of APs at the times $\left\{t_{i}^{l}\right\}$, which we write as $(i=1,2, \ldots N)$ :

$$
S_{i}(t)=\sum_{l}^{\mathrm{spk}} \delta\left(t-t_{i}^{l}\right) .
$$

Its mean firing rate $(\nu)$ is defined as the mean number of spikes per unit 
A

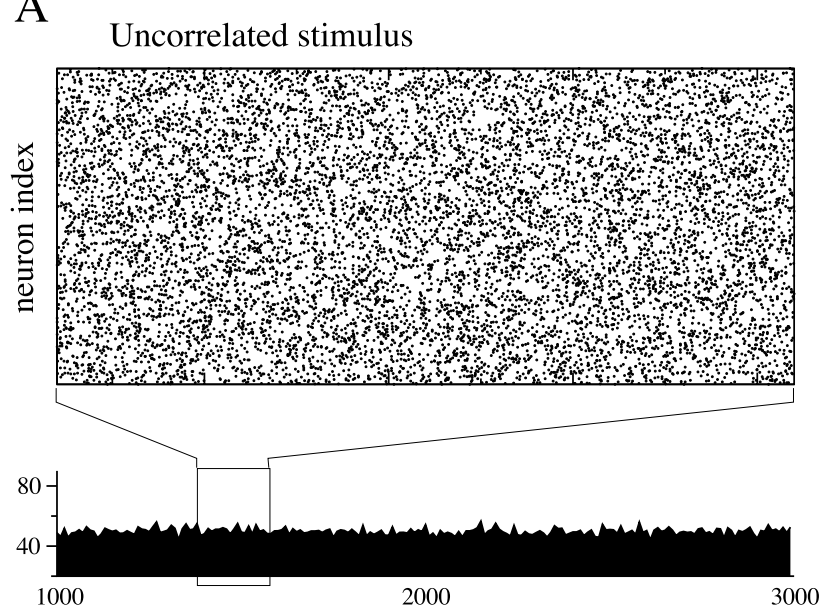

C
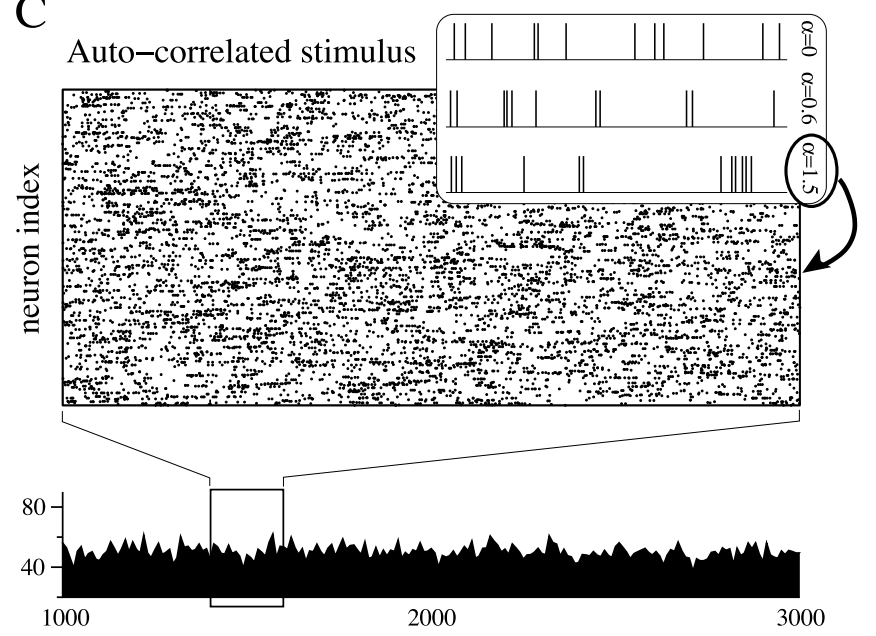

B

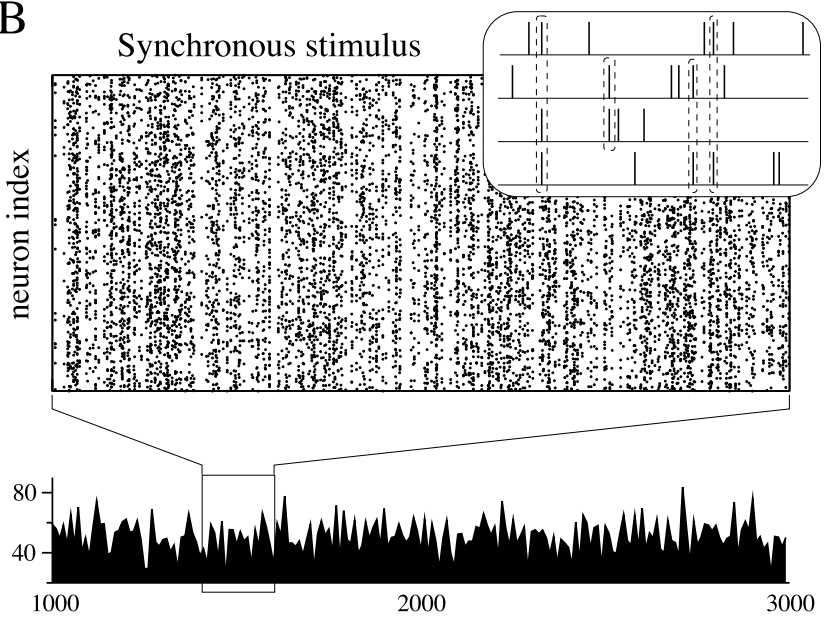

$\mathrm{D}$

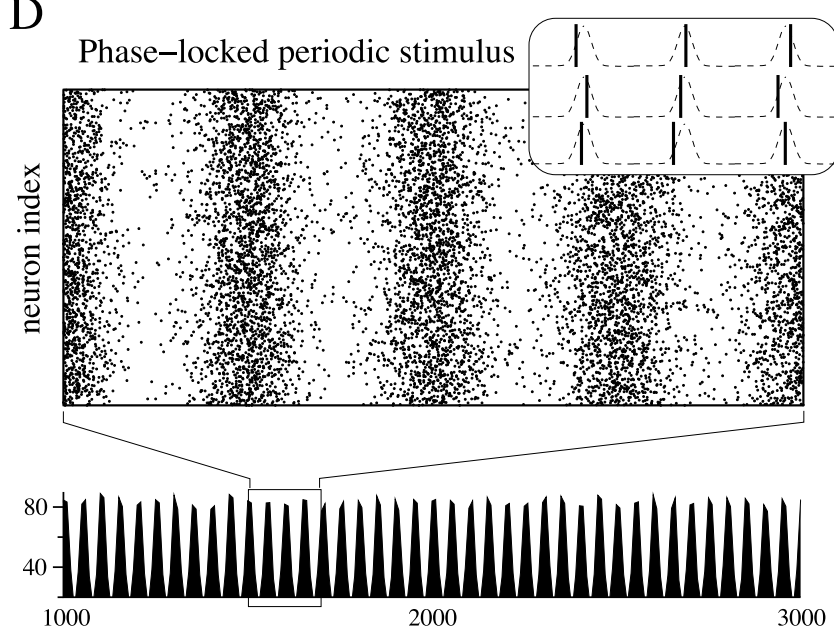

Figure 2. Spike rastergrams of the presynaptic population for the four stimuli studied. $A$, The uncorrelated stimulus lacks any kind of spatiotemporal structure and produces a very homogeneous rastergram. $\boldsymbol{B}$, The synchronous stimulus has a clear spatial structure, because some spikes from different cells fall aligned in time (inset), but it lacks any temporal structure. $\boldsymbol{C}$, The autocorrelated stimulus presents a temporal structure appreciable as horizontal filaments. The inset illustrates three rasters from a single neuron firing with different autocorrelation magnitude: as $\alpha$ increases, the spikes tend to cluster in bursts. $\boldsymbol{D}$, The phase-locked periodic stimulus displays both temporal and spatial correlations. The inset shows rasters from three cells. The common probability distribution function of spike occurrence is shown as dashed lines. The global instantaneous activity (below each raster) was obtained from a single trial using a bin size $d t=10 \mathrm{~ms}$. Thus, it exhibits the variability perceived by the postsynaptic neuron in the spike count in a time window of the size of its membrane time constant (i.e., $\tau_{m}=10 \mathrm{~ms}$ ). These four examples show that spike correlations increase this variability enormously. Parameters are as follows: $N=10,000, \nu=5 \mathrm{~Hz}, \rho=0.002(\boldsymbol{B}), \alpha=1.5(\boldsymbol{C}), f=40 \mathrm{~Hz}$, and $\Sigma=4 \mathrm{~ms}$ (D).

time. Cross-correlations are quantified in terms of the correlation between two spikes of different spike trains $(i \neq j)$,

$$
C_{i j}\left(t, t^{\prime}\right)=\left\langle S_{i}(t) S_{j}\left(t^{\prime}\right)\right\rangle-\left\langle S_{i}(t)\right\rangle\left\langle S_{j}\left(t^{\prime}\right)\right\rangle,
$$

the brackets indicating average over trials, whereas a similar expression is used to define the autocorrelation, which measures the degree of correlation between two spikes of the same spike train,

$$
A_{i}\left(t, t^{\prime}\right)=\left\langle S_{i}(t) S_{i}\left(t^{\prime}\right)\right\rangle-\left\langle S_{i}(t)\right\rangle\left\langle S_{i}\left(t^{\prime}\right)\right\rangle .
$$

Uncorrelated stimuli. This stimulus is built under the assumption of complete independence between any two spikes (regardless of whether they are fired by the same or different cells). In practice, the stimulus consists of a population of presynaptic neurons firing independent Poisson trains with the same constant rate, $\nu$. We will use these stimuli to probe the synaptic model and the effects of varying different synaptic parameters; the elicited neuron response will serve as a control condition with which to compare the responses to correlated stimuli.

Synchronous stimuli. The synchronous stimulus represents the simplest example of instantaneous cooperative behavior between cells. To isolate the effect of spatial correlations from temporal correlations, we consider individual trains without autocorrelations (i.e., they all follow a Poisson statistics with the same rate). The different trains, however, are not generated independently, but they have a certain degree of synchrony, which means that each cell, beyond discharges occurring synchronously by chance, emits a fraction of its spikes at the same time as some other cells (Fig. $2 B$, inset). This is quantified in terms of the crosscorrelation between the spike trains of two presynaptic cells, which takes the following form:

$$
C_{i j}\left(t, t^{\prime}\right)=\nu \rho \delta\left(t-t^{\prime}\right) .
$$

The correlation coefficient $(\rho)$ gives the probability above chance that, given that fiber $i$ produced a spike at time $t$, fiber $j$ produces another spike at the same time. It quantifies with values between 0 and 1 the strength of the cross-correlations. It is 0 when all of the presynaptic neurons fire independently, and it is 1 when they fire replicas of essentially the same spike train.

To numerically generate the synchronous stimulus, we first generated a Poisson "mother" train with rate $\nu / \rho$. Next, each individual train was built as a thinned copy of the mother train from which some spikes, randomly picked, were eliminated with a probability of $1-\rho$ per spike. 
Autocorrelated stimulus. This stimulus is a simple example of cooperative behavior between spikes from the same cell. We chose a stimulus with positive temporal correlations between pairs of spikes from the same cell but not between spikes from different presynaptic trains, which has a simple numerical implementation. More specifically, we generated independent renewal trains with exponential autocorrelations [for details, see de la Rocha et al. (2002)]:

$$
A_{i}\left(t, t^{\prime}\right)=\nu \delta\left(t-t^{\prime}\right)+\nu \alpha \frac{e^{\left(t-t^{\prime}\right) / \tau_{c}}}{\tau_{c}},
$$

and without cross-correlations: $C_{i j}\left(t, t^{\prime}\right)=0(i \neq j)$. The magnitude of the autocorrelations is measured by the dimensionless parameter $\alpha$, which intuitively sets the excess of probability of, given one spike, finding another one within a time range $\tau_{c}$. Notice that $\alpha$ can be varied keeping the firing rate $\nu$ fixed. The inset in Figure $2 C$ shows three examples of spike trains generated with the same rate $\nu=10 \mathrm{~Hz}$, the same correlation range $\tau_{c}=2 \mathrm{~ms}$, but different $\alpha$ values: 0 (which is a Poisson process), 0.6 , and 1.5. It is clear that, for larger values of $\alpha$, the APs cluster in bursts made of more spikes (although their exact number varies from burst to burst). The interval between consecutive spikes within a burst is of the order of $\tau_{c}$.

Phase-locked periodic activity. As a last example, we combined a simple periodic temporal structure with a spatial organization in which the cells coordinate their firing by phase-locking their spikes to an external oscillation of frequency $f$. Individual spike trains are constructed in the following way: each cell emits one AP at the same temporal phase of the oscillation, but with a certain jitter. The value of the jitter of every spike is drawn from a Gaussian distribution of deviation $\Sigma$, truncated on the sides at a half-period distance (to prevent the tails of consecutive Gaussians from overlapping). The parameter $\Sigma$ sets the precision of the phaselocking of each neuron, and its value is kept fixed as the oscillation frequency is varied. This pattern of spikes, which represents the output activity of a network that oscillates rhythmically (Buzsaki and Draguhn, 2004), is illustrated in Figure 2 D.

\section{Synaptic current}

The activity of the $N$ neurons of the presynaptic population gives rise to the stimulus excitatory current $I_{\text {stim }}(t)$. Each AP can produce at most the release of $M$ vesicles. The release from a single vesicle is modeled as an instantaneous pulse of current of size $J$. Thus, the transformations of the train of APs into a sequence of instant current pulses is formulated as follows :

$$
\sum_{i}^{N} S_{i}(t)=\sum_{i}^{N} \sum_{l}^{\text {spk }} \delta\left(t-t_{i}^{l}\right) \rightarrow I_{\text {stim }}(t)=\sum_{i}^{N} \sum_{n}^{M} J_{i, n} \sum_{k}^{\text {rel }} \delta\left(t-t_{i, n}^{k}\right) .
$$

The sums on the right run over presynaptic neurons $(i=1 \ldots N)$, over common contacts for each presynaptic neuron $(n=1 \ldots M)$, and over the releases produced at each particular contact $(k=1 \ldots$ rel $)$. We ignored the variability in the number of common contacts found in experiments (Gil et al., 1999; Silver et al., 2003), so that all connections are made of the same number of them. On the contrary, we captured the variability of quantum response across contacts by taking the efficacies $J_{i, n}$ randomly distributed with a Gaussian of mean $J$ and coefficient of variation $\Delta=0.2-0.4$ (Gil et al., 1999). In the simulations, we will specify the value of the ratio of the efficacy and the membrane capacitance, $J / C_{m}$, which measures the quantal amplitude (i.e., the mean amplitude of the EPSP produced by the release of a single vesicle) in voltage units.

The background current $I_{\mathrm{bg}}(t)$, which represents the afferent activity

${ }^{a}$ In the numerical analysis, we deal with the variable

$$
I_{d t}\left(t_{k}\right)=\frac{1}{d t} \int_{k d t}^{(k+1) d t} I_{\text {stim }}\left(t^{\prime}\right) d t^{\prime},
$$

which is the integrated current over the $k$ th bin of length $d t=1 \mathrm{~ms}$. Thus, when numerically computing the mean or the SD of the current, or when plotting traces, we will inevitably use $I_{d t}(t)$ from other cells of the network or from different brain areas, is generated by Poisson events activating one excitatory and one inhibitory synapse at constant rates $\nu_{E}$ and $\nu_{I}$, respectively. These rates are of the order of several spikes per millisecond, because they represent the superposition of thousands of presynaptic trains at low cortical spontaneous frequencies (e.g., $\sim 1-2 \mathrm{~Hz}$ ).

The total instantaneous synaptic current reaching the target neuron is the sum $I(t)=I_{\text {stim }}(t)+I_{\mathrm{bg}}(t)$. We will normally be interested in its mean and SD. To evaluate them, we will divide the duration $T$ of a long trial into small bins ( of size $d t$ ), build the histogram of the current, and compute its mean $\mu_{I}$ and deviation $\sigma_{I}$ as follows:

$$
\begin{gathered}
\mu_{I}=\frac{1}{N_{T}} \sum_{\text {bins }}^{N_{T}} I(t) \\
\sigma_{I}^{2}=\frac{1}{N_{T}} \sum_{\text {bins }}^{N_{T}} I(t)^{2}-\mu_{I}^{2},
\end{gathered}
$$

where data are taken starting from a time when the response of the target neuron has reached its stationary state (depending on the input rate the stationary regimen has reached after $0.1-1 \mathrm{~s}$ ).

Without background component, the mean current $\mu_{I}$ has a simple analytical expression, because it is equivalent to the mean total charge entering the cell per unit time: because each of the $N M$ contacts is hit by $\nu$ APs per unit time, and only a fraction $P_{t}$ of them trigger the influx of the quantum charge $J$, we have the following:

$$
\mu_{I}=N M J \nu P_{t} .
$$

The deviation $\sigma_{I}$ measures the magnitude of the "fluctuations" produced by the stochasticity of the incoming spikes and of the synaptic transmission. $^{b}$

The model target neuron and the analysis of the response

We model the target neuron as a simple leaky integrate-and-fire (LIF) unit (Ricciardi, 1977). This model describes the dynamics of the membrane potential $V(t)$ when it is below threshold by the following equation:

$$
C_{m} \frac{d V(t)}{d t}=-g_{L}\left(V(t)-E_{L}\right)+I_{\text {stim }}(t)+I_{\mathrm{bg}}(t) \quad \text { if } V<\theta .
$$

Here, $C_{m}$ is the total membrane capacitance, $g_{L}$ is the leak conductance, and $E_{L}$ is the leak potential. The membrane time constant is $\tau_{m}=C_{m} / g_{L}$. The terms $I_{\text {stim }}(t)$ and $I_{\mathrm{bg}}(t)$ represent the stimulus and background current, respectively (see above). Eq. 11 is used until $V(t)$ reaches the spike generation threshold $\theta$. At that point, an AP is discharged, and the potential $V(t)$ is reset to $H$, where it is held during a refractory time $\tau_{\text {ref }}$. The "current threshold" is defined as the value of the current that depolarizes the neuron exactly at its firing threshold; for the integrate-and-fire neuron, it reads $\theta_{I}=\theta g_{L}$.

In this work, we will deal only with the "stationary" regimen, that is, the response of the neuron will be computed after the transmission probability of its synapses has reached its steady state. We will characterize the response mainly by means of the output firing rate $\left(\nu_{\text {out }}\right)$, defined as the mean number of output APs per unit time. The output firing rate as a function of the afferent rate will be referred to as the "response function."

We will also compute the coefficient of variation of the response interspike intervals, $C V_{\text {out }}$, defined as the ratio between the SD and the mean of the output interspike interval. This is a measure of the variability of the output trains, which is 0 for purely periodic trains, equals 1 for Poisson inputs, and is usually $>1$ for bursty spike trains. For the case of a phase-locked stimulus, we will also compute the response vector strength, $V S_{\text {out }}$, which measures the degree of phase-locking of spike responses varying between 0 , for no temporal alignment, and 1 , for perfect phase-locking (Goldberg and Brown, 1969). 


\section{Parameter values}

Unless specified otherwise, we will use the following parameter values: integrate-and-fire model, $E_{L}=0 \mathrm{mV}, C_{m}=300 \mathrm{pF}, \tau_{m}=C_{m} / g_{L}=$ $10 \mathrm{~ms}, \tau_{\text {ref }}=2 \mathrm{~ms}, \theta=15-9 \mathrm{mV}, H=10-6$ $\mathrm{mV}$; synaptic model, $\tau_{v} / N_{0}=600 \mathrm{~ms}, U=$ $0.75, J / C_{m}=0.25 \mathrm{mV}, N_{0}=1-8$; background, $\nu_{E}=3.7$ spikes $/ \mathrm{ms}, \nu_{I}=1.2$ spikes $/ \mathrm{ms}, J_{E} / C_{m}=$ $0.25 \mathrm{mV}, J_{I} / C_{m}=-0.35 \mathrm{mV}$. The total number of synaptic functional contacts $N \times M$ equals 2000.

\section{Simulations}

Because the synaptic current is made of instantaneous pulses of current formally modeled as Dirac $\delta$ functions (Eq. 7), the evolution of the potential (Eq. 11) can be solved in the simulations exactly [i.e., the numerical method used was not approximative (e.g., Runge-Kutta), but it was an exact integration]. The response of the neuron was simulated from three to five trials of 40-100 s, obtaining statistical errors for the output variables of the size of the symbols used in the plots. All simulations were performed on a PC running under SUSE Linux. All graphs were made using the plotting tools Grace and Xfig.

\section{Results}

We will start by introducing the "causal" relationship that, in our model, exists between depression and the FDR. It will be shown that, in this regimen, capturing the synaptic stochasticity in the model of STD has an enormous impact on the response behavior of the target cell. This will be illustrated by probing two different models of STD with uncorrelated poissonian stimuli. The response here will serve as a control condition to compare the response to correlated stimuli analyzed later. The number of functional contacts will also be proved to be very important in shaping the response function.

\section{The fluctuation-driven regimen}

One of the most outstanding effects of STD is that the mean stationary EPSP amplitude decreases with the afferent spike rate $\nu$, behaving as $1 / \nu$ for large enough presynaptic rate (Abbott et al., 1997; Tsodyks and Markram, 1997) (Fig. 3A). The mean current $\mu_{I}$ is proportional to the product of the mean EPSP and $\nu$ (Eq. 10) and therefore saturates as $\nu$ increases (Fig. $3 B$ ). The average membrane potential, which can be approximated by $\langle V\rangle \approx \mu_{I} / g_{L}$, is also upper bounded and its maximum value is given by the following:

$$
\langle V\rangle_{\max } \approx \frac{\mu_{\max }}{g_{L}}=\frac{N M J \tau_{m}}{C_{m} \tau_{v}},
$$

where $\mu_{\max }$ is simply obtained by substituting in Eq. 10 the rate of releases $\nu P_{t}$ by its maximum value, given by the rate at which vesicles recover, $1 / \tau_{v}$. The question now is whether this upper bound can constrain the cell to be, regardless of the presynaptic rate, in the so-called "fluctuation-driven regimen" (Gerstein and Mandelbrot, 1964; Calvin and Stevens, 1968; Shadlen and Newsome, 1994). In the FDR, the mean
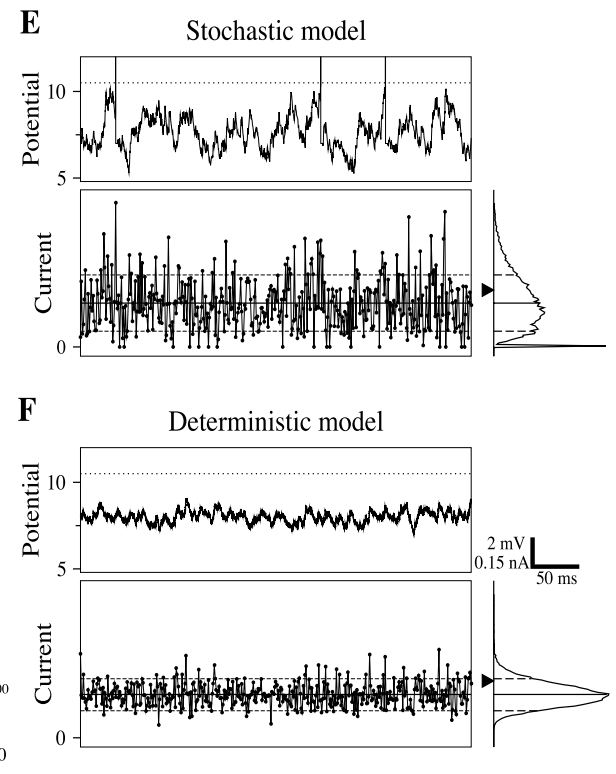

$v(\mathrm{~Hz})$

Deterministic Stochastic

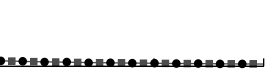

0

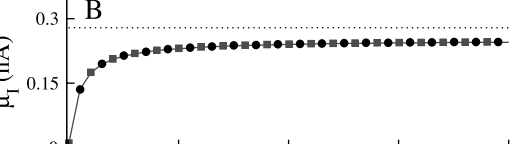

Figure 3. Comparison between the stochastic and the deterministic models of STD. The two models (stochastic, squares; deterministic, circles) are compared in the case of asynchronous Poisson input spike trains. $A$, The transmission probability, which model: in the deterministic model, it shows a non-monotonic behavior and takes substantially lower values

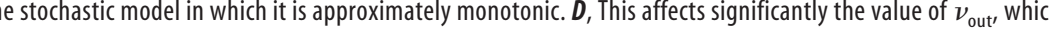
suprathreshold regimen (the threshold was reduced to $\theta=7.4 \mathrm{mV}$ ), where $\sigma_{l}$ does not determine the response behavior and $10.5 \mathrm{mV}$ (for the traces), and $H=6 \mathrm{mV}$. The background activity was not included. The rest of the parameters are as specified in Materials and Methods. spk/sec, Spikes/second.

depolarization must be lower than the threshold, a condition that is formulated as follows:

$$
\langle V\rangle_{\max }<\theta,
$$

which after substituting $\langle V\rangle_{\max }$ by the expression above can be reformulated as follows:

$$
N<\theta \frac{C_{m} \tau_{v}}{J M \tau_{m}} .
$$

Assigning some realistic values to the parameters on the righthand side allows us to estimate the number of presynaptic neurons required to violate this inequality. Taking $\theta=15 \mathrm{mV}$, $M J / C_{m}=1 \mathrm{mV}$ (which is a representative value of the nondepressed EPSP size), $\tau_{v}=500 \mathrm{~ms}$, and $\tau_{m}=10 \mathrm{~ms}$, we obtain that, if $N<750$, the condition given in Eq. 13 is satisfied. We confirmed this result by going through the same analysis with a conductance-based LIF model obtaining a boundary of $N<$ 600 (see Appendix). Although the total number of afferent connections received by a cortical neuron exceeds these figures (Braitenberg and Schüz, 1991), estimations of the size of what can be considered a population of neurons encoding the 
same information fall below this number [e.g., around a hundred (Shadlen and Newsome, 1998)]. ${ }^{c}$

Depression therefore implies that a neuron receiving an input signal from a few hundred cells will work in the FDR. This is true in the stationary state, that is, after the short transient interval required to depress the synapses. In the FDR, current fluctuations play a fundamental role in driving the response of the neuron, because the membrane potential reaches $\theta$ only on the arrival of positive fluctuations of $I(t)$. That is why their correct modeling takes special importance in this regimen.

\section{Current fluctuations in the deterministic and stochastic models of STD}

To show the relevance of the stochastic nature of the synaptic transmission, we compare the current deviation and the neuron response produced by two models of STD, namely, the stochastic model of vesicle turnover described in Materials and Methods and the widely used deterministic model of "averaged" synaptic responses (Abbott et al., 1997; Tsodyks and Markram, 1997). When $N_{0}=1$, both models give, by construction, the same mean current (Fig. 3B), but they lead to current fluctuations of different magnitude and different behavior as a function of the rate (Fig. $3 C$ ). Because the deterministic model was proposed to fit synaptic responses averaged over trials (Tsodyks and Markram, 1997; Varela et al., 1997), it lacks the trial-to-trial variability observed in synaptic transmission (Stevens and Wang, 1994), and therefore the fluctuations it generates are smaller than those produced by the stochastic model, particularly at high input rates (Fig. 3C). Specifically, in the stochastic model, a smaller probability of transmission, $P_{t}$, increases the fraction of failure spikes. In contrast, $P_{t}$ equals the normalized mean EPSC in the deterministic model, and its decrease produces just smaller PSC amplitudes (something that contradicts the quantal hypothesis) without filtering any of the incoming spikes. At very large rates, the current in the deterministic model is composed of a series of PSCs of negligible size, closely spaced in time. This synaptic current has almost no fluctuations and resembles, in the limit of large input rate, an injection of constant current. This subtle difference makes $\sigma_{I}$ grow monotonically with $\nu$ toward its saturation value in the stochastic model, whereas in the deterministic model $\sigma_{I}$ shows a non-monotonic behavior tending to 0 at high input rates (Fig. 3C).

Because the target cell is in the FDR, the amplitude of the afferent fluctuations mainly determines its response function. Thus, $\nu_{\text {out }}$ is much larger and saturates monotonically in the stochastic model, whereas in the deterministic model it inherits the non-monotonic behavior of $\sigma_{I}$ and vanishes at high rates (Fig. $3 D$ ). Figure 3, $E$ and $F$, illustrates traces generated by both models at $\nu=60 \mathrm{~Hz}$. Both produce the same mean depolarization, but the smaller magnitude of the current fluctuations in the deterministic model generates smaller fluctuations of the potential, making the neuron fire at a lower rate.

If the mean current saturates above threshold (i.e., the suprathreshold regimen), the two models give similar rates, because in this case the response is basically determined by the mean current drive and the fluctuations play a secondary role (Fig. $3 D$, inset).

The stochastic model seems, in summary, a more appropriate

An interesting example to look at is the average number of thalamocortical connections a cortical cell receives. Models of V1 usually consider between 15 and 200 connections (Chance et al., 1998; Troyer et al., 1998; Shelley et al., 2002), whereas in the barrel cortex estimates are in the order of a few tens (Kyriazi and Simons, 1993). model to investigate the response in the FDR, given that the deterministic model leads to the artifact of vanishing fluctuations at high $\nu$, an effect that would contaminate the analysis performed in what follows.

\section{Effect of the synaptic parameters $M$ and $N_{0}$ on the neuron response}

We turn now to investigate the relevance of the size of the RP $\left(N_{0}\right)$ and the number of functional contacts per presynaptic neuron $(M)$ in the response to uncorrelated stimuli. Studying the response produced by synapses with several functional contacts will serve us to understand the impact of spike synchrony analyzed later. This happens because multiple functional contacts trivially give rise to the synchronous release of vesicles, which will produce the same effect qualitatively as a synchronous stimulus.

The size of the RP is a controversial issue. Different experiments in hippocampal slices have led to different values: whereas Stevens and collaborators report values between 2 and 20 with mean $\left\langle N_{0}\right\rangle \sim 5$ (Dobrunz and Stevens, 1997; Murthy et al., 2001), other groups have reported that the immediately releasable pool, or "primed" pool, has a size close to 1 (Hanse and Gustafsson, 2001). Theoretical analysis of paired-pulse depression in cortical neurons has reached the same conclusion, namely, that in some synapses there must be a last "bottleneck" pool that only holds around one vesicle (Matveev and Wang, 2000b). ${ }^{d}$ For a fair comparison of synapses with different $N_{0}$, the recovery time was adjusted to keep the ratio $\tau_{v} / N_{0}$ fixed (see Materials and Methods) (Fig. 1C).

Regarding the number of common contacts, it is a widely variable quantity across brain areas. An average number from the somatosensory cortex lies around 5 for pyramidal cells (Markram et al., 1997; Silver et al., 2003) and 17 for GABAergic interneurons (Gupta et al., 2000). Among noncortical synapses, one can find examples in which $M$ rises up to 15-20 in the spinal cord (Walmsley, 1991), 20 in the cerebellum (Pedroarena and Schwarz, 2003), and $>1000$ in the famous end-bulbs of Held (Held, 1893; Schneggenburger et al., 2002). For a fair comparison of cases with different $M$ values in the following analysis, the total number of contacts, $N \times M$, is kept fixed (Fig. $1 A, B$ ).

The comparison of the transmission probability (Fig. $4 A$ ) and the mean number of readily releasable vesicles (Fig. $4 B$ ) for two different RP sizes $\left(N_{0}=1\right.$ and $\left.N_{0}=4\right)$ reveals that these two synaptic types differ only at low frequencies $(\nu<15 \mathrm{~Hz})$. In this range, synapses with larger $N_{0}$ have a larger $P_{t}$ and, on average, have more vesicles ready for release. However, as the input rate increases, more spikes reach the synapses per unit time and the $\mathrm{RP}$ becomes more depleted. Both the transmission probability and the mean number of releasable vesicles tend to 0 as $1 / \nu$ (dashed lines in Fig. $4 A, B$ represent the fit $1 / \nu$ ). At these high rates, synapses with different $N_{0}$ are indistinguishable. In other words, a synapse with $N_{0}>1$ behaves as a synapse with a single vesicle $\mathrm{RP}$, but with a recovery time constant $\tau_{v} / N_{0}$ (Matveev and Wang, 2000a; de la Rocha, 2003). This can be understood by noting that, at high input rates, the RP is normally empty and sometimes has at most one docked vesicle. Thus, if for instance $N_{0}=2$, after complete depletion of the RP, the replenishment of one vesicle can come from the recovery of any of the two vacan-

${ }^{d}$ The particular case $N_{0}=1$ also has a desirable property: in this situation, the stochastic model is directly consistent with the phenomenological deterministic model based on "averaged responses" (Fuhrmann et al., 2002). In other words, averaging the synaptic responses produced by the stochastic model with $N_{0}=10$ ver repetitions of the same presynaptic spike train yields mean EPSP amplitudes that are well fitted with the deterministic model. 

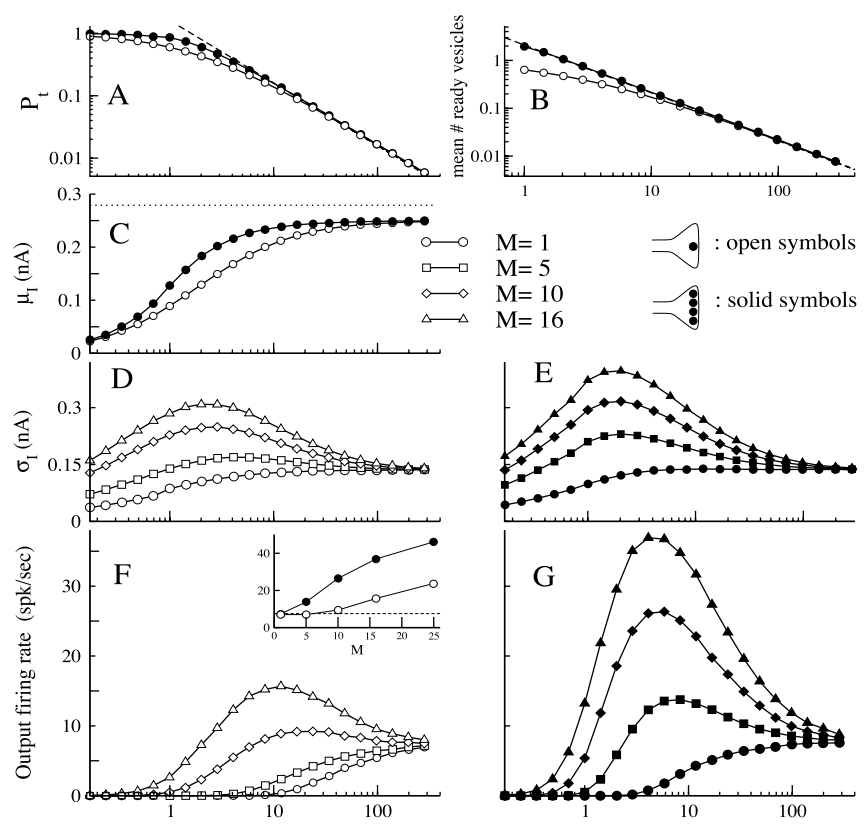

Input firing rate $(\mathrm{spk} / \mathrm{sec})$

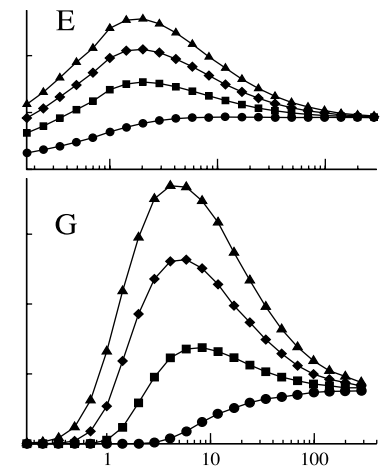

Input firing rate $(\mathrm{spk} / \mathrm{sec})$

Figure 4. Impact of the RP size $N_{0}$ and the number of common contacts $M$ on the response. $\boldsymbol{A}, \boldsymbol{B}$, The transmission probability $(\boldsymbol{A})$ and the mean number of ready-releasable vesicles $(\boldsymbol{B})$ of synapses with $N_{0}=1$ (open) and 4 (solid) is plotted against $\nu$. Regardless of the value of $N_{0}$, both quantities follow the same $1 / \nu$ dependence at high $\nu$ (dashed lines are the fit $1 / \nu$ ). $\mathbf{C}$, The mean current, which is independent of $M$, saturates to the same value for both $N_{0}$ values. Before saturation, it is larger in the case $N_{0}=4$ because of the larger value of $P_{t}$. The dotted line represents $\theta_{l}$ and indicates that the cell is in the FDR. $\boldsymbol{D}, \boldsymbol{E}$, The current deviation is plotted for several values of $M$ in the two cases $N_{0}=1(\boldsymbol{D})$ and $N_{0}=4(\boldsymbol{E})$. In both cases, synapses with more common contacts generate a larger $\sigma_{l}$, but at high rates the deviation converges to a common value regardless of the number $M . \boldsymbol{F}, \boldsymbol{G}$, The response firing rate acquires a resonant behavior when the number of functional contacts is large enough (for $M \geq 5$ if $N_{0}=4$ and for $M \geq 10$ if $N_{0}=1$ ). The asymptotic value of $\nu_{\text {out }}$ does not vary with $M$, meaning that at a large input rate, different $M$ values give rise to the same response. $\boldsymbol{F}$, inset, shows the maximum $\nu_{\text {out }}$ versus $M$. The dashed line indicates the asymptotic value of $\nu_{\text {out }}$ (i.e., the $\nu_{\text {out }}$ obtained when $\nu \rightarrow \infty)$. Parameters are as in Figure $3 A-D$ except that $\theta=10.6 \mathrm{mV}$ and $H=9 \mathrm{mV}$. Background is not included. Notice the log scale used in the horizontal axes. spk/sec, Spikes/second.

cies, so that it can be viewed as a single vesicle process with a twice as large recovery rate.

The mean current produced by synapses with $N_{0}=1$ and $N_{0}=4$ is plotted in Figure $4 C$. In both cases, vesicle depletion produces the saturation of $\mu_{I}$ at high input rates. Because, as it was just shown, at high rates these synaptic types are indistinguishable, it is expected that they produce the same current statistics. Before the mean saturates, synapses with larger $N_{0}$ generate a larger $\mu_{I}$ because the transmission probability is larger. With the parameters chosen here, $\mu_{I}$ saturates below current threshold (Fig. 4C, dotted line), which implies that the target cell works in the FDR at all input rates.

The current SD is non-monotonic

As opposed to the mean current, which depends only on the total number of functional contacts, $N \times M$, current fluctuations are very sensitive to the particular value of $M$ (Fig. $4 D, E)$ : when the synaptic connections have several common contacts, the variability of the current is larger because the PSCs are made of 1, or $2, \ldots$, or $M$ quantum events. In contrast, if $M=1$, the PSCs are always unitary and only the random trigger of two simultaneous releases by different presynaptic cells may occasionally generate larger PSCs. This can be seen in Figure $4, D$ and $E$, where $\sigma_{I}$ is plotted for several $M$ values (for $N_{0}=1$ and $N_{0}=4$, respectively): at low and moderate input rates, $M$ sets the gain of the fluctuations so that larger $M$ values produce larger $I$ values. However, as $\nu$ increases, $\sigma_{I}$ converges to a value independent of $M$. This asymptotic convergence, in combination with the increase at low rates, endows $\sigma_{I}$ with a non-monotonic behavior when $M>1$, regardless of the value of $N_{0}$.

Why does the effect of having $M>1$ vanish at high input rates? Figure 5 illustrates the explanation: top and bottom diagrams illustrate the sequence of releases (bars) and recovery intervals (horizontal bands) produced at five common contacts by a sequence of afferent spikes (top bars) reaching the presynaptic terminals at $\nu=2 \mathrm{~Hz}$ (diagram A) and $\nu=40 \mathrm{~Hz}$ (diagram B). Because $N_{0}=1$, when a spike arrives during the recovery interval of a contact, it produces a failure (dots) and otherwise it triggers a release (i.e., $U=1$ ). At the arrival of the first spike, the five contacts are fully recovered, and the first AP triggers the synchronous release of five vesicles. In the case in which $\nu=2 \mathrm{~Hz}$, each contact has time to recover before the next spike arrives, and thus the synchrony in the releases across the $M$ common contacts is maintained. The PSCs generated at this input rate are large, because they are normally composed of three or four quantum events, and therefore they generate large current fluctuations. In contrast, when $\nu=40 \mathrm{~Hz}$, the second AP arriving at the presynaptic terminal finds all contacts depleted and the next "unitary" release does not occur until one contact is eventually replenished. Because the replenishment of vesicles occurs independently at each contact, releases occur asynchronously producing mostly unitary PSCs that give rise to smaller current fluctuations. In conclusion, at high rates the releases produced at common contacts become effectively asynchronous, because the transmission probability becomes so small that, on arrival of an AP, at most one of the contacts succeeds in releasing transmitter.

\section{Non-monotonic behavior of the neuron response}

Because the target cell has been set in the FDR, this nonmonotonic modulation of the current fluctuations has a great impact on the output firing rate (Fig. $4 F, G$ ): the response function inherits the non-monotonic behavior of $\sigma_{I}$ and converges at high input rates to a value independent of the number of functional contacts. Because $M$ sets the gain of the fluctuations for low and moderate values of $\nu$, it consequently also sets the gain of the response rate.

The non-monotonicity is more prominent for synapses with $N_{0}=4$ than for those with $N_{0}=1$. This occurs mainly as a consequence of the larger mean current for $N_{0}=4$ (Fig. 4 C) but also because the magnitude of the current fluctuations is a little larger in that case (Fig. 4, compare $D, E$ ). Figure $4 F$, inset, shows the maximum output rate plotted versus $M$ for those two cases: one sees that $N_{0}$ sets the sensitivity of the response to variations of the gain parameter $M$. For this reason, the non-monotonic shape of $\nu_{\text {out }}$ appears clearly for all $M>4$ when $N_{0}=4$, whereas a much larger value of $M(M>9)$ is required when $N_{0}=1$.

A graphical explanation of why the response function acquires a resonant shape can be viewed in Figure 6. The figure shows the afferent spikes (top rasters), transmitter releases (bottom rasters), synaptic current (top traces), and membrane potential (bottom traces) produced by a presynaptic population with a rather large $M=16$, which serves to illustrate the effect more clearly. These variables were monitored at low $(\nu=5 \mathrm{~Hz})$ and high $(\nu=100 \mathrm{~Hz})$ input rate. At $\nu=5 \mathrm{~Hz}$, the fewer APs per time unit trigger synchronous releases at common contacts (Fig. 6A, bottom raster); because we set $N_{0}=4$, these synchronous releases were approximately preserved even in the case that incoming 

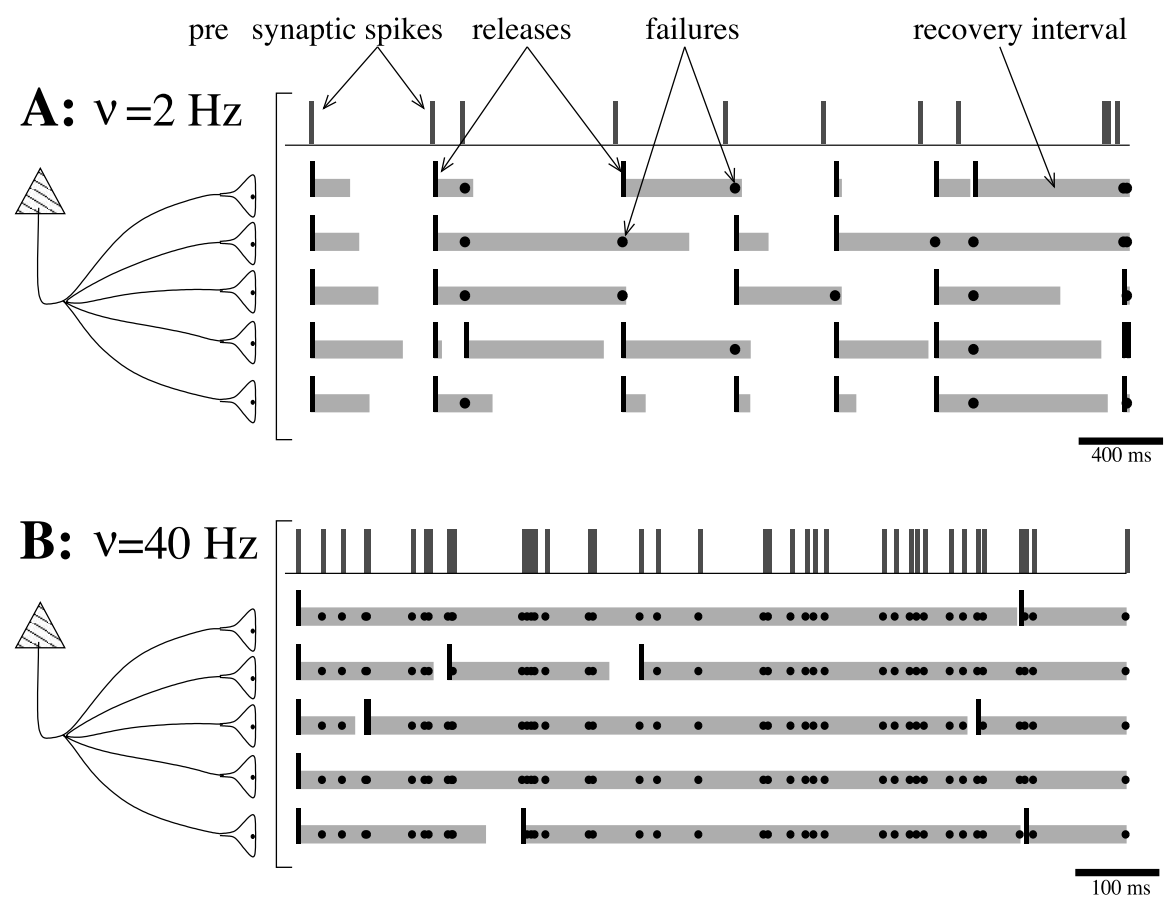

Figure 5. Desynchronization of releases produced at $M$ common functional contacts. $\boldsymbol{A}, \boldsymbol{B}$, In each diagram, top bars represent the input spike train for $\nu=2$ and $40 \mathrm{~Hz}$, respectively. Below, each row represents the releases (bars) produced by those spikes in each of the $M=5$ synaptic contacts established by a presynaptic cell. The releases are followed by the vesicle recovery intervals (horizontal boxes), something that occurs randomly and independently across contacts. During these intervals, spikes fail to trigger any response (solid dots); otherwise they produce a release (i.e., we set $N_{0}=1$ and $U=1$ ). It is clear that, at low rates $(\nu=$ 2 in $A$ ), the releases occurring at the common contacts are well synchronized, whereas, at higher rates $(\nu=40 \mathrm{~Hz}$ in $B$ ), they occur almost independently. Note the different time scale of each diagram.
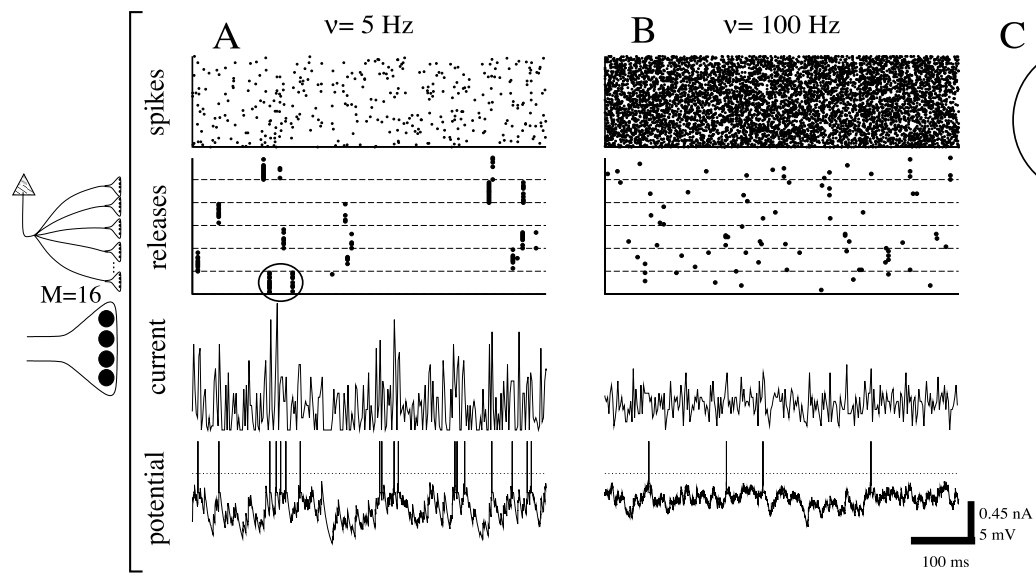

Figure 6. Current and potential traces produced by synapses with many common contacts. In $\boldsymbol{A}$ and $\boldsymbol{B}$, the rasters represent spikes from the presynaptic population (top) and releases triggered by six presynaptic cells (bottom). The grid dashed lines group together releases from common contacts. The bottom traces show the synaptic current (top) and the membrane potential (bottom) of the postsynaptic cell. The dotted line represents the threshold. $\boldsymbol{A}$ and $\boldsymbol{B}$ represent low and high input rates, respectively. $\boldsymbol{A}$ Because $M=16$, the releases triggered by common contacts look like vertical bars when they occur synchronously at low input rate. Releases inside the circle are redrawn at a larger scale in $\boldsymbol{C}$. Synchronous releases generate very large fluctuations of the synaptic current, which make the membrane potential fluctuate strongly, giving rise to a large output rate. $\boldsymbol{B}$, When the input rate is $\nu=100 \mathrm{~Hz}$, releases from common contacts do not show any synchronous structure and produce current with a smaller variability. The membrane potential shows smaller fluctuations, and because the target cell operates in the FDR, they give rise to a lower output rate. Parameters are $M=16$ and $N_{0}=4$, and the rest are as in Figure $3 A-D$.

spikes came closely spaced in time (Fig. 6C). Because of the large PSCs produced, the potential could reach threshold very frequently, giving rise to a large spiking response. At $\nu=100 \mathrm{~Hz}$, the structure of common contacts is not perceptible in the release raster (Fig. $6 B$, bottom raster). The PSCs generated, which are

synchronous releases. now more, are mostly unitary and the fluctuations of the current are severely reduced. The potential, which has a mean that now lies closer to the threshold, does not fluctuate in the strong manner that it did at low input rate, and therefore it reaches $\theta$ fewer times. When the input rate is very low $(\nu<2 \mathrm{~Hz})$, although the magnitude of the PSCs can be very large, they are so few that the output rate is also low (traces not shown).

\section{Synchronous stimulus}

We will now discuss a correlated stimulus in which the presynaptic neurons fire synchronous Poisson trains with the same constant rate $\nu$ and a correlation coefficient $\rho$ (see Materials and Methods). We will show that the impact of synchrony across afferent APs resembles very much that of synchronous releases produced by common synaptic contacts, examined above.

As a general rule, modifying the correlations between presynaptic trains, while keeping their rate fixed, does not alter the "mean" synaptic current but may change the current deviation substantially. As a consequence, any information conveyed by the spatiotemporal correlations of the input is transmitted by the fluctuations of the current but not by the mean drive. For reliable static synapses, synchronizing the afferent spikes increases the probability of synchronous releases, which give rise to PSPs composed of several unitary events. This increases the magnitude of the fluctuations (Shadlen and Newsome, 1998; Salinas and Sejnowski, 2000; Moreno et al., 2002b), something that generally increases the output rate, particularly in the FDR (Salinas and Sejnowski, 2000; Moreno et al., 2002b)..$^{e}$ For synapses showing STD, synchrony increases $\sigma_{I}$ too, although in a nonuniform way with $\nu$ (Fig. $7 A$ ): for low rates, the increase is larger than for high rates generating a nonmonotonic behavior of $\sigma_{I}$ as a function of $\nu$ (Moreno et al., 2002a). This occurs because the impact of synchrony is weighted by the transmission probability $P_{t}$, which decreases with $\nu$ (Fig. 3A) (J. de la Rocha, R. Moreno-Bote, and N. Parga, unpublished observations). In other words, at high rates, the input synchrony does not affect the response, because it becomes rather unlikely that the simultaneous arrival of APs triggers the corresponding 
The non-monotonic behavior of $\sigma_{I}$ is again inherited by $\nu_{\text {out }}$, which displays a tuned dependence on $\nu$ with a "preferred" presynaptic rate, $v_{p}$. The preferred rate depends on the synaptic parameters $\tau_{v}, U$, and $N_{0}$ (data not shown). However, it remains almost unaltered to variations of $\rho$ within a wide range of values of the correlation coefficient $\left(\rho \sim 0.01-0.1\right.$ for $N_{0}=$ 1). Furthermore, $\rho$ sets the amplitude of the tuning curve acting as a "gain control parameter," for low and moderate input rates, within the range $\rho \sim 0.01-0.06$ (for $N_{0}=1$, see Fig. $7 B$ ). The maximum response initially follows an approximately linear relationship with $\rho$ and later saturates (Fig. $7 C$ ).

In Figure $7 D$, we lowered the threshold while keeping the rest of the parameters fixed, so that the cell sits in the suprathreshold regimen. As expected, the response no longer has a resonant shape but increases monotonically for all correlation values. Increasing the degree of synchrony increases the rate, especially at low $\nu$ at which $\mu_{I}<\theta_{I}$ (i.e., the cell is still in the FDR), and depression is not very prominent. This change, however, does not represent an increase of the gain, as it approximately does in the subthreshold regimen (Fig. $7 B$ ). Hereafter, we will therefore restrict the analysis to the FDR and will come back to the general case in Discussion.

The gain modulation observed in the FDR and attributable to the afferent spike correlations has important consequences in the transmission of information carried by the rate $\nu$ to the postsynaptic cell. In the absence of synchrony, $\sigma_{I}$ is barely modulated by the input rate (Fig. $7 \mathrm{~A}$, full circles), so that the information can be transmitted by $\mu_{I}$ only in the range of low and moderate $\nu$ values, because at high presynaptic rates, $\mu_{I}$ undergoes very little modulation (Fig. 7A, solid line). Synchronizing the afferent APs acts as a gain for $\sigma_{I}$ (Fig. $7 A$ ), which because of depression may acquire a prominent non-monotonic behavior. Thus, when the mean current is saturated, the current fluctuations become the main carrier of information, enabling the modulation of the response (1) within a larger dynamical range of the response and (2) for a wider range of $\nu$ (Fig. $7 A, B$ ). For instance, for $\rho=0.05$, the response undergoes a $\sim 10 \mathrm{~Hz}$ variation in the range $\nu=20-100 \mathrm{~Hz}$ (Fig. $7 B$ ), whereas $\mu_{I}$ barely varies in this range (Fig. $7 A$, solid line).

Figure $7 E$ shows traces of the current and the membrane potential at three presynaptic rates, namely, at $1 \mathrm{~Hz}$, at the preferred frequency $\nu_{p}=9 \mathrm{~Hz}$, and at $80 \mathrm{~Hz}$, for $\rho=0.04$. Apart from the synchronized stimulus, the target cell receives a constant background activity (see Materials and Methods). As the rate increases, the positive fluctuations in the current (produced by synchronous releases) occur more often but are smaller, and the Figure $3 A-D$. spk/sec, Spikes/second.

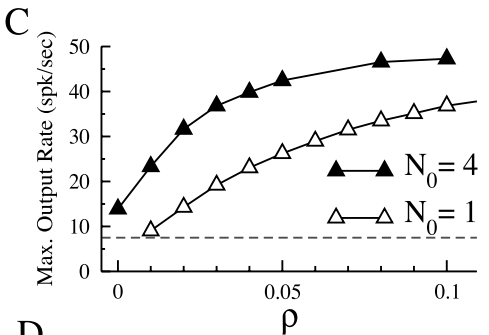

$\mathrm{D}$
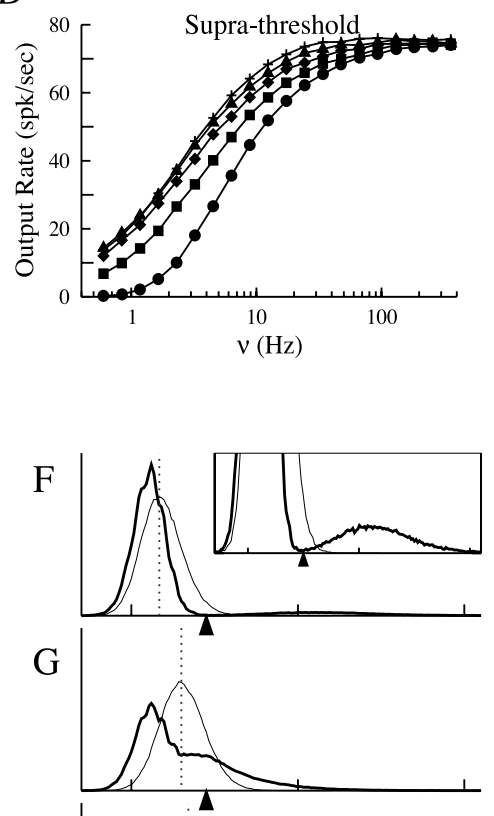

$\mathrm{H}$

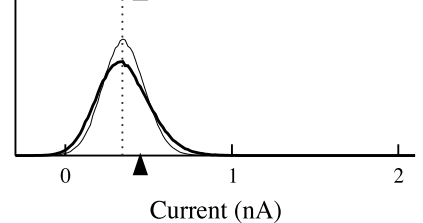

Figure 7. Effect of STD using a synchronous stimulus. The synaptic current and the neuron response are analyzed when the presynaptic Poisson trains are synchronized with correlation coefficient $\rho$ (Fig. 2B). A, B, Current deviation $\sigma_{l}$ and output rate versus $\nu$ for several degrees of synchrony. Synchrony endows the current fluctuations with a resonant behavior, which is inherited by the response rate. The degree of synchrony $\rho$ has almost no effect on the position of the maximum, but it finely sets the gain of asymptotic value of $\nu_{\text {out }}$. $\boldsymbol{D}$, The threshold was lowered to $\theta=11 \mathrm{mV}$ to analyze the response in the suprathreshold regimen. $\boldsymbol{E}$, Current (top) and potential (bottom) traces for three input frequencies with $\rho=0.04$. Horizontal dotted line represents threshold. Inset, A magnification in the $y$-axis of the same plot in which the tail cannot be perceived. The vertical dotted line represents $\mu_{1}$ arrowheads represent the position of $\theta_{l}$. Parameters are as follows: $\theta=15 \mathrm{mV}, H=10 \mathrm{mV}, M=5$, and $N_{0}=1$. Whit and black arrowheads in $A$ signal $\theta_{l}$ for the subthreshold and suprathreshold regimens. Background is included. The rest are as in

mean current increases, taking the mean depolarization closer to the threshold. Therefore, the neuron responds maximally at $\nu_{p}=$ $9 \mathrm{~Hz}$, because the current reaches a compromise between large fluctuations and large mean (note that the preferred frequency in Fig. $7 B$ does not produce the maximal fluctuations in Fig. $7 A$ ). The histograms of the current at these three rate values are shown in Figure 7, $F-H$, respectively (thick lines), along with the asynchronous case, $\rho=0$ (thin lines), drawn for comparison. At each frequency, both histograms have the same mean (dotted lines), which always falls below $\theta_{I}$ (arrowheads in the figure), but at $\nu=$ 1 and $9 \mathrm{~Hz}$ they differ substantially (Fig. $7 F, G$ ). In these two cases, synchronous releases skew the distribution and increase its deviation, something that increases the output rate significantly. At higher rate $(\nu=80 \mathrm{~Hz})$, the skewness, which is the signature of 

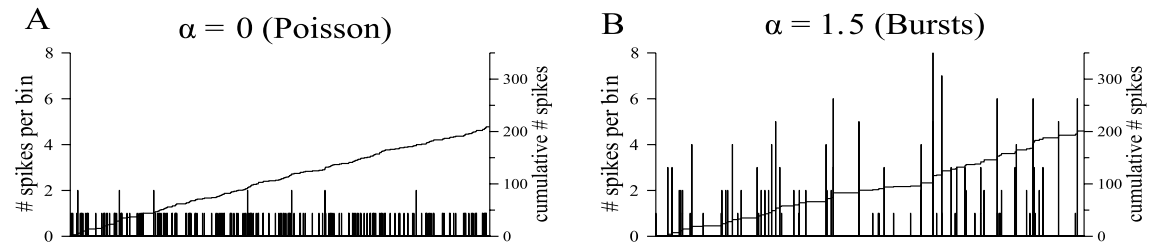

$\mathrm{C}$
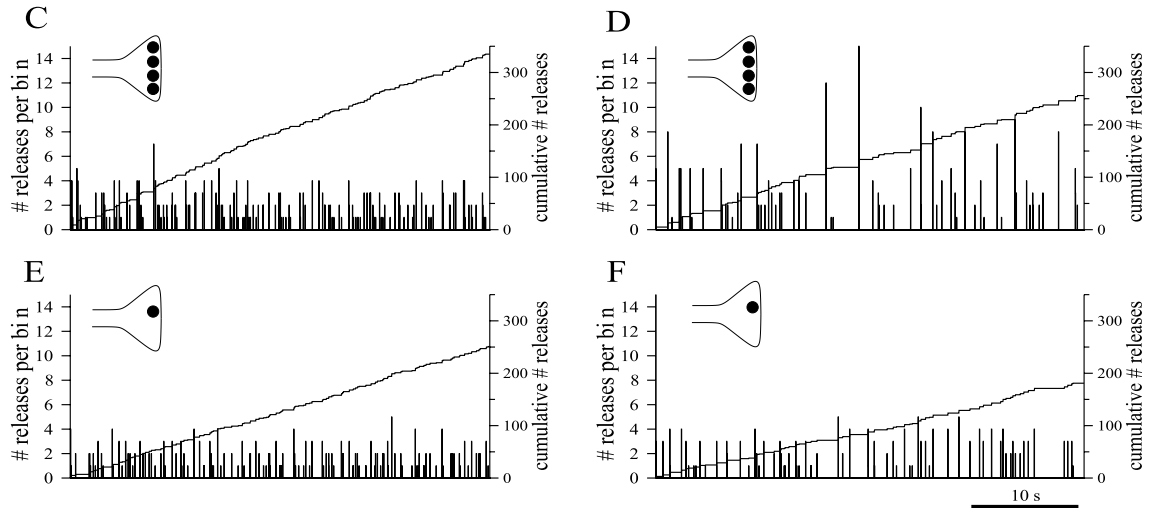

Figure 8. Autocorrelated spike trains. Simulation examples of fluctuations produced in the spike count $(\boldsymbol{A}, \boldsymbol{B})$ and release count $\left(\boldsymbol{C}, \boldsymbol{D}, N_{0}=4 ; \boldsymbol{E}, \boldsymbol{F}, N_{0}=1\right)$ by a single spike train with and without autocorrelations (left and right panels, respectively) with a fixed rate of $\nu=5 \mathrm{~Hz}$. The spike count (or release count) per bin is plotted versus time on the left axes. Monotonically increasing lines represent the cumulative spike count (or release count) on the right axes. $\boldsymbol{A}, \boldsymbol{B}$, The Poisson input normally generates a single spike per bin and occasionally two. In contrast, the case of $\alpha=1.5$ produces large positive fluctuations in the spike count because of the presence of bursts. $\boldsymbol{C}-\boldsymbol{F}$, These large spike count fluctuations are transmitted to the release count only if $N_{0}=4(\boldsymbol{D})$, and they are essentially filtered if $N_{0}=1(\boldsymbol{F})$. When $M=5$, the release count fluctuations are amplified. Concerning the total release count produced, it can be observed that the train with autocorrelations elicits fewer releases than the Poisson train regardless of $N_{0}$ (compare $\boldsymbol{D}$ with $\boldsymbol{C}$, and $\boldsymbol{F}$ with $\boldsymbol{E}$ ). Bin size $d t=10 \mathrm{~ms}$.

synchronous releases, disappears and the two distributions become similar.

To better understand the consequences that synchrony and STD may have in the transmission of information, we went beyond the analysis of the output rate and studied higher-order statistics of the response. In this direction, we performed a simulation of two target neurons receiving APs from nonoverlapping subpopulations, which are part of the same population of synchronously firing cells. We then computed the output correlation coefficient of the target pair, $\rho_{\text {out }}$, which because of the afferent correlations is different from 0 . The observed general trend is that, as synapses depress with an increasing afferent rate, the correlation coefficient $\rho_{\text {out }}$ drops off to 0 (data not shown). This is completely consistent with what we just saw, namely, that as synapses become more unreliable the impact of input synchrony vanishes, affecting the output firing rate but also weakening the ability of the presynaptic population to generate additional synchrony in the output ensemble of cells. We will go over the implications of this result in Discussion.

To summarize, the impact of synchronized incoming spikes is very similar to that of synaptic connections with several common contacts, but the functional implications are very different, because $\rho$ can be a dynamical variable, whereas $M$ was a fixed parameter. Synchronized stimuli increase the current fluctuation gain at low and moderate input rates, something that enhances the gain of the non-monotonic response function.

\section{Autocorrelated stimulus}

Neurons in cortical and subcortical areas often display positive autocorrelations in their spike times (Bair et al., 1994; Dan et al., 1996; Baddeley et al., 1997; Goldman et al., 2002), meaning that the emission of a spike by a presynaptic neuron increases the probability of observing a new discharge of the same neuron over a short time interval (e.g., $\sim 10-20 \mathrm{~ms}$ ). In other words, spikes are not homogeneously spread in time, but they tend to come in clusters. A typical case of short-range positive autocorrelations is the emission of bursts observed in many different areas (Lisman, 1997; Sherman, 2001; Krahe and Gabbiani, 2004). We used a particular instance of input statistics that produces spike trains with short-range positive exponential autocorrelations (see Materials and Methods). The magnitude of the autocorrelations is set up by a parameter $\alpha$, which yields a Poisson process for $\alpha=0$ and trains with a bursty temporal structure for $\alpha \geq 1.5$ ("bursts" composed of a variable number of spikes with mean $\sim 4-5$ ). Examples of these spike trains for several values of $\alpha$ can be seen in Figure 2C, inset.

For nondepressing synapses, positive autocorrelations have the same qualitative effect as spatial cross-correlations (Moreno et al., 2002b): the impact produced by incoming clusters of APs does not depend on whether the spikes within the cluster come from the same or from different presynaptic cells. ${ }^{f}$ This simple principle seems not to be applicable when synaptic transmission depends on the presynaptic activity. With facilitating synapses, for instance, a cluster of spikes coming from the same or from different cells, would not produce the same depolarization: only if they are emitted by the same presynaptic neuron, the synapse facilitates and gives rise to a larger depolarization. However, we will see that, under the choice of certain parameter values of the stochastic model, bursts of spikes may produce qualitatively the same effect as the synchronous activity considered before, although with a quantitatively smaller overall impact.

Clustering the incoming spikes can increase the fluctuations of the synaptic current, because it may induce the temporal summation of consecutive PSCs elicited by the spikes within the burst. This increase of the fluctuations is analyzed at the level of the release statistics in Figure 8, where we compare two inputs with the same rate but different correlation magnitudes: $\alpha=0$ (Poisson) and $\alpha=1.5$ (bursts). Figure 8, $A$ and $B$, shows the number of spikes falling in bins of size $\tau_{m}$ versus time. ${ }^{g}$ As expected, the correlated case produces large positive fluctuations because of the existence of bursts and the uncorrelated case looks very homogeneous, producing one or no spikes per bin and only occasionally two. Figure 8, $C$ and $D$, depicts the number of "releases" produced by those spike trains when the RP size is $N_{0}=4$ and $M=5$. The bursty input still produces larger fluctuations than the Poisson stimulus. Notice that, because every AP can produce from zero up to five releases, the variability of the releases seems amplified with respect to that of the spike trains.

Figure $8, E$ and $F$, also shows a sequence of releases but for

${ }^{f}$ This simple idea makes sense only if the spatial dimension of the postsynaptic cell can be ignored. ${ }^{g}$ Bin size was taken equal to $\tau_{m}$, because this is the longest time scale at which fluctuations in the number of spikes are perceived by the target model neuron (Moreno et al., 2002b). Smaller bin sizes (e.g., $1 \mathrm{~ms}$ ) would not capture the clustered structure of the input. 
synapses with $N_{0}=1$. In contrast to the case $N_{0}=4$, the large fluctuations of the bursty input have been severely filtered out so that now both bursty and Poisson inputs produce fluctuations of similar magnitude. This difference can be easily understood: bursts produced after a long silent interval might find the RP fully replenished. Then, if $N_{0}=1$, the burst can release at most one vesicle per contact, whereas if $N_{0}>1$, the same burst can release up to $N_{0}$ vesicles. This explains that, only in the case $N_{0}>1$ and when preceded by a long enough silent interval, a cluster of APs can be faithfully translated into a significant fluctuation in the number of releases. At high rates, the required long silent periods never occur. The model with $N_{0}>1$ becomes effectively a model with $N_{0}=1$ (as explained previously) (Fig. $4 \mathrm{~B}$ ), and the fluctuations coming from the bursts are again filtered.

In addition, clustering the incoming spikes decreases the mean release count, because a larger fraction of APs find the synapses depleted. The monotonically increasing lines in Figure 8 represent the cumulative number of events (spikes or releases) produced by Poisson and bursty inputs (scale on the right vertical axes). Because both stimuli have the same rate $(\nu=5 \mathrm{~Hz})$, the total number of spikes is about the same ( $\sim 200$ spikes) (Fig. $8 A, B)$. However, the total release count is smaller for the bursty stimulus regardless of the value of $N_{0}$ (Fig. $8 C-F$ ).

Positive input autocorrelations, therefore, may alter the response of the cell in opposite directions depending on the relative impact of these two effects, namely (1) the decrease of the mean release count which implies a decrease of $\mu_{I}$ and occurs for any size $N_{0}$ (Fig. $9 A, B$ ); (2) the increase of the fluctuation of the release count, which implies an increase of $\sigma_{I}$ and crucially depends on the value of $N_{0}$ : when $N_{0}=1$ the bursty stimulus generates fluctuations with a similar overall magnitude as the Poisson stimulus (Fig. 9C), whereas if $N_{0}>1, \sigma_{I}$ increases substantially at low and intermediate input rates (Fig. 9D). As a consequence, only when $N_{0}>1(\text { and } M \geq 5)^{h}$, the increase in $\sigma_{I}$ yields to an increase of $\nu_{\text {out }}$ with $\alpha$, in that way enhancing the resonant behavior obtained previously with Poisson input (Fig. $9 F$ ). If $N_{0}=1$, it is the decrease in $\mu_{I}$ that dictates the decrease of the response (Fig. 9E). The behavior at high input rate is expected: synapses saturate and, for any $\alpha$, both $\mu_{I}$ and $\sigma_{I}$ (and in turn $\left.\nu_{\text {out }}\right)$ converge to the value given by the uncorrelated stimulus.

Figure 9, $G$ and $H$, shows the response $C V_{\text {out }}$ for the two cases $N_{0}=1$ and $N_{0}=4$. As explained, when $N_{0}=1$, autocorrelations are severely filtered so that the $C V_{\text {out }}$ remains unaffected when $\alpha$ is increased. In contrast, when $N_{0}=4$, bursty trains produce slightly larger $C V_{\text {out }}$ values if the synapses are not very depressed, which in this example means $\nu<30 \mathrm{~Hz}$. Figure $9 \mathrm{H}$, inset, shows this sensitivity of the $C V_{\text {out }}$ to $\alpha$ at two values of the input rate, $\nu=10$ and $40 \mathrm{~Hz}$. Although, for $\nu<30 \mathrm{~Hz}$, bursts of spikes increase $C V_{\text {out }}$, the impact is rather small if one considers, for instance, that autocorrelated trains with $\alpha=1.5$ have themselves an input $C V=2$ and produce a maximum $C V_{\text {out }} \approx 1.2$.

Spike and release rasters together with current and potential traces neatly illustrate this effect (Fig. 10). Setting $M=5$ for both types of synapse, a Poisson and a bursty stimulus were compared at a moderate input rate, $\nu=5 \mathrm{~Hz}$. When $N_{0}=1$, the bursts of spikes are filtered by depletion of the synapses, a fact evident in the nonbursty structure of the raster of releases compared with the corresponding raster of autocorrelated spikes (Fig. $10 \mathrm{~B}$, bot-

${ }^{h}$ The number of common contacts turns out to be a crucial parameter as well. Unless $M \geq 5$, the increase $\sigma$ produced by the autocorrelations is too little to overcome the decrease in $\mu_{l}$ and, including autocorrelation, leads to an decrease in the response even in the case $N_{0}>1$ (data not shown).

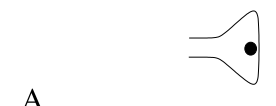

A
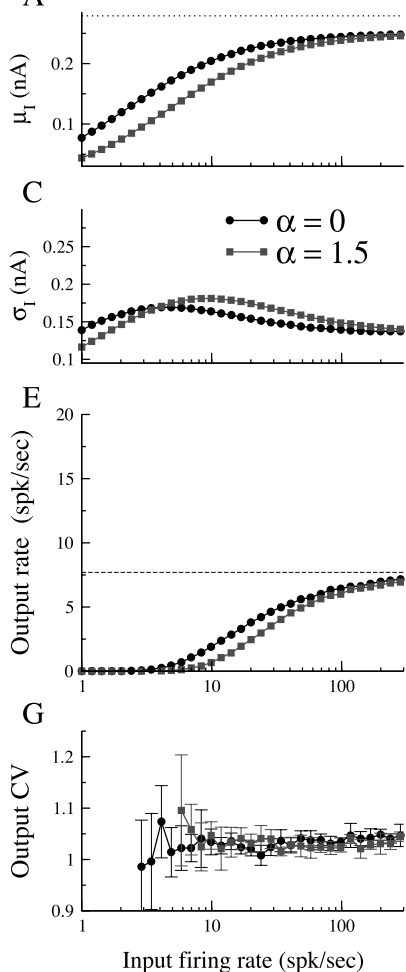

Input firing rate $(\mathrm{spk} / \mathrm{sec})$

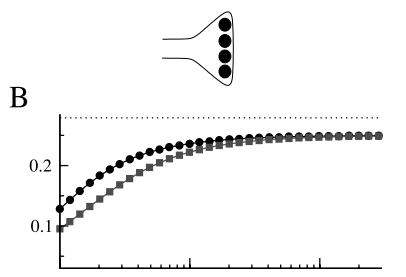

D
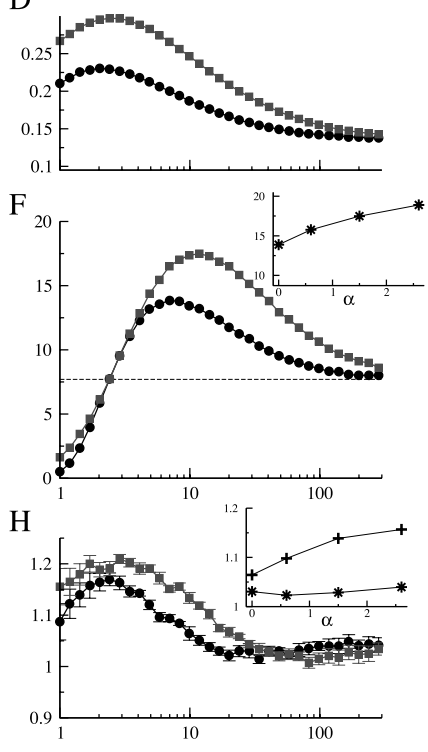

Input firing rate $(\mathrm{spk} / \mathrm{sec})$
Figure 9. Impact of autocorrelated stimuli using synapses with different $N_{0}$. The response to uncorrelated $(\alpha=0)$ and autocorrelated stimuli $(\alpha=1.5)$ is analyzed for $M=5$ and two values of the RP size: $N_{0}=1$ (left) and $N_{0}=4$ (right). $\boldsymbol{A}, \boldsymbol{B}$, The bursty stimulus produces a smaller $\mu_{l}$ in both cases. The dotted line shows $\theta_{l}, \boldsymbol{C}, \boldsymbol{D}$, The impact of autocorrelations in $\sigma_{l}$ is different in each type of synapse: for $N_{0}=1$, bursts do not increase the overall magnitude of the fluctuations ( $\left(C\right.$. In contrast, with $N_{0}=4$, the autocorrelations have a big impact, increasing $\sigma_{l,}$ because spike bursts can trigger release bursts. At high $\nu$, autocorrelations have no effect on either $\mu_{l}$ or $\sigma_{l}, \boldsymbol{E}, \boldsymbol{F}$, As a consequence, if $N_{0}=4$, autocorrelations enhance the resonant response rate $(\boldsymbol{F})$, whereas if $N_{0}=1$, the response decreases slightly $(\boldsymbol{E})$. Dashed lines indicate the asymptotic value of $\nu_{\text {out }} . \boldsymbol{F}$, Inset, Maximum $\nu_{\text {out }}$ versus $\alpha$ for $N_{0}=4 . \boldsymbol{G}, \boldsymbol{H}$, The response $C V_{\text {out }}$ shows a slight increase with the input autocorrelations only if $N_{0}=4$ and $\nu<30 \mathrm{~Hz}$. $\boldsymbol{H}$, Inset, $\mathrm{CV}_{\text {out }}$ versus $\alpha$ for $N_{0}=4$ at $\nu=10(+)$ and $40 \mathrm{~Hz}(*)$. Parameters are as follows: $\theta=$ $10.6 \mathrm{mV}, H=9 \mathrm{mV}, d t=10 \mathrm{~ms}$. The rest are as in Figure $3 A-D$. Legend in Capplies to all of the plots. spk/sec, Spikes/second.

tom and top rasters). Because of this efficient filtering of bursts, fewer releases are triggered in comparison with the Poisson case (Fig. 10A, $B$, compare bottom rasters) and the current trace shows a reduction of its mean with barely any variation in the fluctuations (Fig. 10 A, B, compare top traces). As a result, the response firing rate is smaller for the bursty stimulus than for the Poisson input (Fig. 10 A, B, bottom traces). In contrast, the example with $N_{0}=4$ shows that an afferent burst does usually elicit several consecutive releases (a thinned version of the original spike burst) (Fig. $10 \mathrm{D}$, bottom raster). This leads to an increase of the current fluctuations that, despite the decrease of $\mu_{I}$, yields an increase of the response firing rate (Fig. $10 C, D$, traces).

\section{Phase-locked spike trains}

We saw that, in the saturation regimen, synaptic depression may filter out the input synchrony between presynaptic trains and the positive autocorrelations of individual trains. We now show that this effect also occurs when cross-correlations are generated by a common drive. With nondepressing synapses, "signalcorrelations" increase the output rate (Salinas and Sejnowski, 

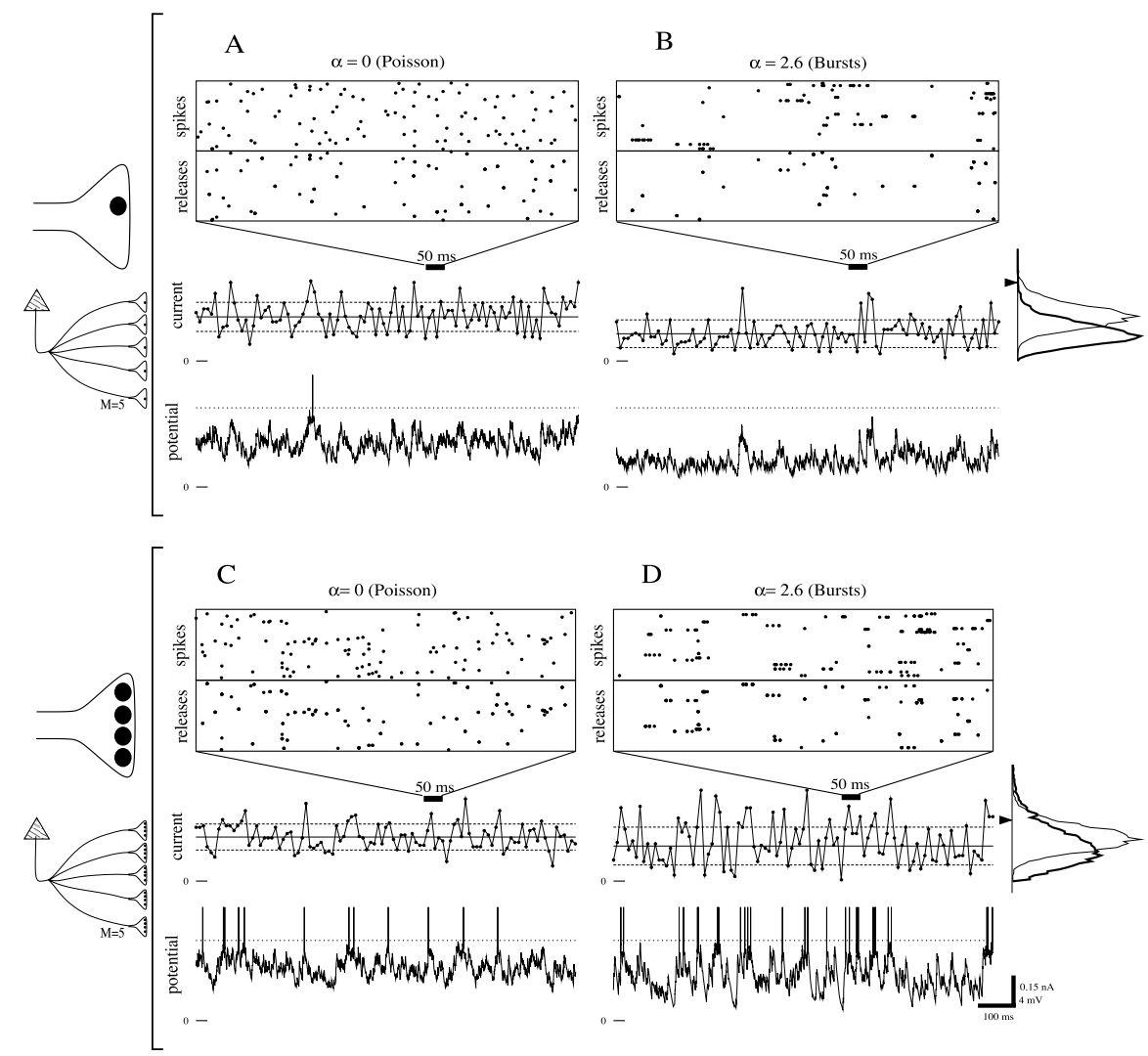

Figure 10. Current and potential traces produced by autocorrelated stimuli using synapses with different $N_{0}$ values. In $\boldsymbol{A}-\boldsymbol{D}$, the rasters represent spikes from the presynaptic population (top) and the releases triggered by successful spikes (bottom). The traces show the synaptic current (top trace; histograms on the right) and the membrane potential of the postsynaptic cell (bottom trace). Horizontal lines represent $\mu_{l}$ (solid), $\mu_{l} \pm \sigma_{l}$ (dashed), and threshold potential (dotted). $\boldsymbol{A}$ and $\boldsymbol{C}(\boldsymbol{B}, \boldsymbol{D})$ represent Poisson (autocorrelated) stimuli. $\boldsymbol{A}, \boldsymbol{B}$, If $N_{0}=1$, the spike bursts in the autocorrelated stimulus are not transmitted as bursts of releases, so that more spikes fail to release transmitter in comparison with noncorrelated input (compare their release rasters). This means that the autocorrelations lead to a substantial reduction of $\mu_{l}$ with little variation of $\sigma_{l}$ (see histograms in which thin line is $\alpha=$ 0 and thick line is $\alpha=2.6$ ). As a consequence, the target cell is less depolarized and its firing rate decreases (see membrane traces). $\boldsymbol{C}, \boldsymbol{D}$, In contrast, when $N_{0}=4$, a burst of spikes may trigger a thinned burst of releases, which provokes an increase of the current fluctuations that, despite the decrease of $\mu_{\prime \prime}$ makes the output rate increase (see membrane traces). Parameters are as follows: $\nu=5 \mathrm{~Hz}, M=5, \alpha=0(\boldsymbol{A}, \boldsymbol{C})$, and $\alpha=2.6(\boldsymbol{B}, \boldsymbol{D})$. The current was binned using $d t=10 \mathrm{~ms}$. The rest of the parameters are as in Figure 9.

2000) (but see also Grande et al., 2004), which happens just because a coordinated population of neurons can produce larger depolarizations than a population of independent neurons. Let us then consider a simple configuration in which each presynaptic neuron fires, locked to a periodic signal of frequency $f$, one spike per cycle. The precision of the spike timing is limited by a fixed Gaussian jitter of SD $\Sigma$ (for details, see Materials and Methods).

Figure 11 shows the response of the target neuron as a function of the oscillation frequency for four values of $\Sigma$ along with a case in which each cell fires phase-locked to the same signal but at a different phase (incoherent mode). As expected, coordinating the firing across afferent trains in a coherent manner gives a larger response, but only for low and intermediate input frequencies. As with the two previous stimuli, in the coherent condition, $\nu_{\text {out }}$ shows a resonant behavior, with a preferred frequency $f_{p}$ (Fig. $11 A$ ). Beyond $f_{p}$, responses decrease sharply until a critical value $f_{c}$ is reached (e.g., $f_{c} \sim 23 \mathrm{~Hz}$ for $\Sigma=10 \mathrm{~ms}$ ), at which the response coincides with that of the incoherent case. Beyond $f_{c}$, the output rate in the two modes are indistinguishable.

Figure $11 B$ illustrates the input (dashed lines, $V S_{\text {in }}$ ) and output (symbols, $V S_{\text {out }}$ ) vector strengths, a measure that quantifies between 0 and 1 the precision of the phase-locking of the spikes. Because the parameter $\Sigma$ is held constant as $f$ varies, the jitter relative to the cycle length increases with the frequency, except for the case $\Sigma=0 \mathrm{~ms}$. This produces the monotonic decrease of $V S_{\text {in }}$ if $\Sigma>0$. Remarkably, in these three cases, $V S_{\text {out }}$ is always larger than $V S_{\text {in }}$, although eventually it also drops to 0 as the frequency increases.

The non-monotonic behavior of the response function occurs because, for depressing synapses, $P_{t}$ decreases with $f$ so that releases in a given synaptic contact occur for a smaller fraction of cycles, a fact that decreases the correlations between the releases across contacts. In addition, if $\Sigma>0$, correlations become weaker, because the relative jitter increases. For $f>f_{c}$, the correlations are so small that they do not affect the response rate of the cell. However, the output spikes still carry information about the input signal, as can be observed, in that $V S_{\text {out }}$ becomes 0 at a frequency significantly higher than $f_{c}$ (Fig. $11 B)$. This is particularly true when $\Sigma=0$, at which the input vector strength is always 1 , and as a consequence $V S_{\text {out }} \sim 1$ in a very large range of $f$ (up to $1 \mathrm{kHz}$ ).

Because for non-zero jitter, $V S_{\text {in }}$ decreases with the frequency, one might be tempted to attribute the non-monotonic shape of $\nu_{\text {out }}$ to this characteristic of the stimulus or to its combined effect with depression. The nonphysiological case $\Sigma=0$ allows us to isolate the effect of depression from that of the $V S_{\text {in }}$ decrease. Because the non-monotonicity occurs here without decrease in $V S_{\text {in }}$, it becomes clear that the resonant effect is solely attributable to depression and that the decrease of $V S_{\text {in }}$ only affects the position of the maximum.

Finally, it is worth mentioning that the resonant effect is still present in the more general case in which cells fire at every cycle with a certain probability $p<1$ (data not shown).

\section{Generalization of the results}

In the present work, we constrained the analysis to "temporally homogeneous" stimuli. However, it is clear that, in the general case, the variability of the synaptic current registered from a cell would have a large contribution from temporal variations of the afferent signal. In a recent work, we explored the effect of depression over time-varying signals defined by their instantaneous firing rate, finding that, if the synapses work in saturation, the timevarying structure of the input is also severely weakened as it is converted into a synaptic current (J. de la Rocha and N. Parga, unpublished observations). This damping of the afferent structure, which implies a loss of spatiotemporal information, seems therefore to be a general property on synapses whose transmission reliability is substantially decreased as the presynaptic activity increases. This is not an exclusive feature of vesicle depletion; a number of presynaptic mechanisms also yield a decrease of $P_{t}$ : inactivation of release machinery (Hsu et al., 1996), presynaptic 

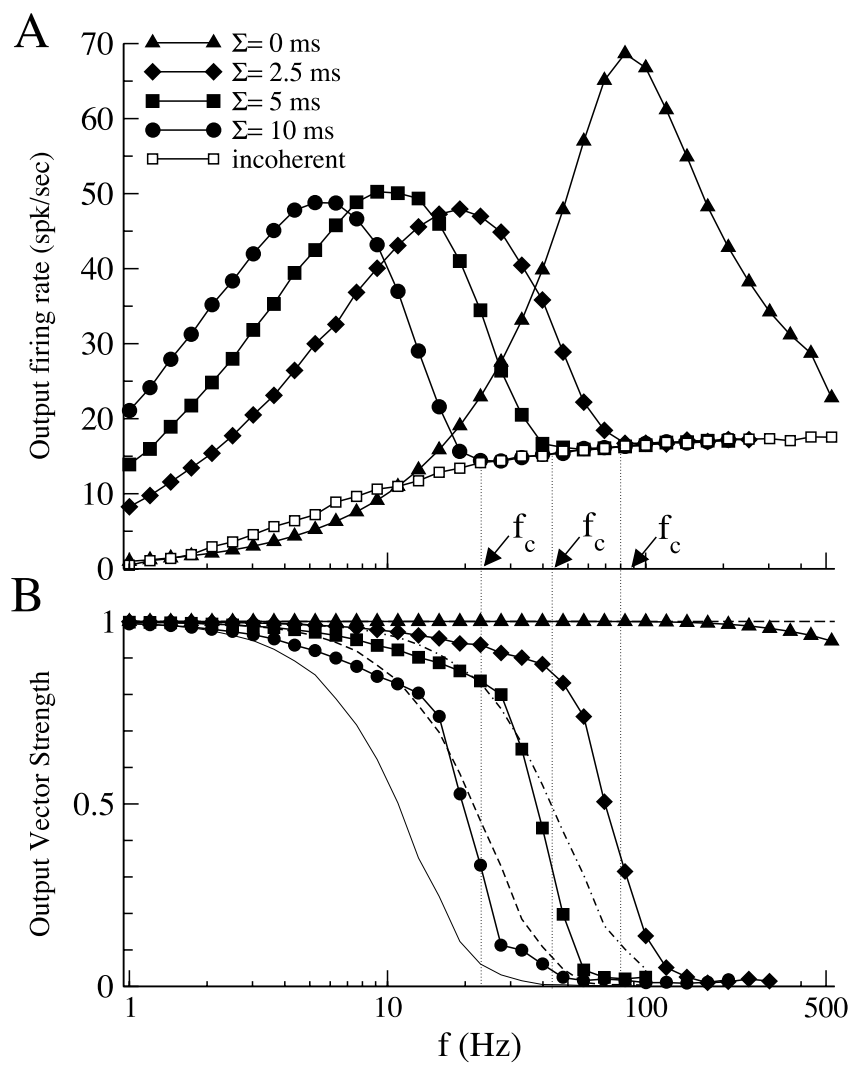

Figure 11. Response to phase-locked periodic stimuli. $\boldsymbol{A}$, The response rate to phase-locked presynaptic trains (Fig. 2D) is shown for four different jitter magnitudes. The response of a case in which the individual phases were randomly chosen (incoherent mode) is represented with open symbols. The coherent input shows a resonant behavior and becomes indistinguishable from the incoherent mode for high enough frequencies (beyond the corresponding $f_{c}$ ). $B, V S_{\text {out }}$ versus $f$ is shown (symbols) along with the input vector strength (solid, $\Sigma=10 \mathrm{~ms}$; dashed, 5 $\mathrm{ms}$; dot-dashed, $2.5 \mathrm{~ms}$; and long-dashed, $0 \mathrm{~ms}$ ). Parameters are as follows: $M=5, \theta=14 \mathrm{mV}$, $H=10 \mathrm{mV}, N_{0}=1$. Background is included. spk/sec, Spikes/second.

inhibition via metabotropic receptors (Davies and Collingridge, 1990; Scanziani et al., 1997), and others (for review, see Zucker and Regehr, 2002). The non-monotonic behavior in $\nu_{\text {out }}$ is achieved if $P_{t}$ decreases at least as $1 / \nu$, something observed in the cases cited above (Matveev and Wang, 2000a) but violated by synapses showing activity-dependent recovery, that is, when $\tau_{v}$ decreases with $\nu$ (Dittman and Regehr, 1998; Stevens and Wesseling, 1998; Wang and Kaczmarek, 1998; Fuhrmann et al., 2004).

If synapses exhibit both depression and facilitation (Thomson, 1997; Markram et al., 1998), the resonance is still present because facilitation does not prevent the synapse from saturating at high $\nu$ because of vesicle depletion (de la Rocha et al., 2004). With facilitation alone, the result would however be very different, namely, that only at high input rates, when the synapses are facilitated, correlations would have a significant impact. The response function would no longer be non-monotonic but would produce superlinear behavior.

We used a somehow detailed model of the presynaptic terminal, but an oversimplified leaky integrate-and-fire neuron model without neither synaptic conductances nor synaptic filters. However, preliminary simulations using an LIF model with synaptic conductances confirm our main results (data not shown). This occurs because the kind of correlations used here change the variance of the total current but do not alter the mean total conductance substantially. Furthermore, depression constrains the increase in the mean total conductance obtained from an increase in the presynaptic rate. This is attributable to the saturation of the release rate, which imposes an upper bound on the mean conductance that an ensemble of cells can generate. In other words, a finite presynaptic population cannot make the effective membrane time constant of a cell increasingly smaller by simply bombarding the cell at a higher rate, because synaptic depression prevents it.

\section{Discussion}

How does STD transform a neural signal conveyed by the coordinated firing of an ensemble of presynaptic cells? STD leads to saturation of the transmitter release process as the input rate $(\nu)$ increases. This implies (1) the saturation of the mean current $\left(\mu_{I}\right)$ and (2) the loss of correlations in the afferent spike trains. If the stimulus has spatiotemporal structure, the combination of these two effects leads to a non-monotonic response function provided that the neuron operates in the FDR.

The transformation of neural signals performed by shortterm plasticity has been the focus of many theoretical analyses in the last years (Abbott et al., 1997; Tsodyks and Markram, 1997; Chance et al., 1998). Depression based on vesicle depletion makes the reliability of the synaptic transmission become a function of the afferent activity. As increasing the number of afferent APs per time unit depletes the readily releasable pool of vesicles, the transmission probability decreases at high input rate as $P_{t} \sim 1 / \nu$ (Abbott et al., 1997; Tsodyks and Markram, 1997; Matveev and Wang, 2000a). This leads to the saturation of the mean synaptic current, which becomes independent of the input rate (Eq. 10). This result seemed to constrain the range of presynaptic rates in which information could be efficiently encoded to low values of this rate (Abbott et al., 1997; Tsodyks and Markram, 1997). This argument, however, ignores that the postsynaptic response is determined not only by the mean synaptic drive but also by the current fluctuations.

This is particularly true in the FDR, a scenario in which the mean membrane potential (without spiking) lies below threshold and only the fluctuations of the current make $V(t)$ reach threshold (Gerstein and Mandelbrot, 1964; Shadlen and Newsome, 1994). This regimen has been usually discussed under the assumption of a "balance" between the excitatory and the inhibitory inputs (Shadlen and Newsome, 1994; van Vreeswijk and Sompolinsky, 1996). When STD is considered, the saturation of the mean current below the current threshold puts the target cell in this regimen, without the need of the balance between excitation and inhibition. This result does not rule out the balance hypothesis as an important aspect underlying the FDR, but it proposes a different complementary mechanism by which a cell may stay in the FDR regardless of the spike rate of the presynaptic population. Thus, the existence of depression alone would keep a network of excitatory cells away from having suprathreshold stable states, and it would set the FDR as the default network regimen. Experimentally, one could distinguish which mechanism underlies the FDR in each case because of their different signatures: whereas a balanced state generally implies a large increase in the total conductance of the cell (Borg-Graham et al., 1998), depression upper-bounds this increase (see Results) and its presence can be better characterized by a decrease in the average EPSP amplitude (Chung et al., 2002).

The existence of the non-monotonic response has several computational implications. First, information can be transmitted to the postsynaptic cell by the current fluctuations, even at high values of the input rate $(20-80 \mathrm{~Hz})$ at which the mean 
synaptic current has saturated previously (Figs. $7 A, B ; 9 B, F$ ). In addition, the cell displays a response function selective to a certain range of frequencies as opposed to the classical notion of a response that increases monotonically with stimulus intensity. This selectivity can be computationally interesting in sensory areas where cells are often tuned and respond maximally to certain stimuli. Whereas the conventional view has been to ascribe response tuning properties to solely circuit mechanisms, our results present a single-cell mechanism, which could cooperate with the circuitry to establish tuning. In the auditory system, for example, cortical cells display a non-monotonic behavior as a function of sound intensity (Phillips and Irvine, 1981; Sutter and Schreiner, 1995), an effect that could be partially replicated with our model by considering that thalamocortical auditory cells increase their rate with sound intensity and fire with a certain degree of synchrony. In our non-monotonic response curves, the preferred rate depends basically on the parameters $U$, and $\tau_{v}$ of the synaptic model (data not shown), which have been found to undergo long-term plasticity (Markram and Tsodyks, 1996). Such longterm changes could enable the neurons to modify their tuning properties.

Whereas depression provides the non-monotonicity, correlations determine the magnitude of this modulation: synchrony, for instance, generates a gain modulation, without the need of network interactions (Fig. 7B) [although considering them would probably sharpen the tuning (Salinas and Abbott, 1996)]. This gives rise to a mechanism of gain control, a phenomenon with functional consequences that have been widely studied (Salinas and Thier, 2000).

Our results also have important implications on the stability of recurrent circuits. Differences in the levels of depression observed in cortical excitatory and inhibitory synapses have been proposed previously to be a mechanism promoting stability (Galarreta and Hestrin, 1998; Varela et al., 1999). Our findings add two different elements to the stability analysis. First, a nonmonotonic response represents a highly efficient way to maintain adequate levels of activity. It prevents positive feedback from becoming a source of instability because a large fluctuation in the network activity always leads to a decrease in the activity in the next integration period. Second, as mentioned before (see Results), depression compromises the efficacy of input synchrony on generating "output" synchrony, because unreliable synapses weaken the overall effect of presynaptic correlations. This would prevent the network from developing epileptiform discharge patterns: a transient partial synchronization would increase the firing rate of the network. This increase would depress the recurrent synapses, thus dampening the recruitment of additional synchrony and preserving the stability of the asynchronous state.

Several recent studies have addressed the general problem of how current fluctuations impact on the neuron response (Chance et al., 2002; Fellous et al., 2003; Kuhn et al., 2004). In vitro experiments have consistently shown that increasing the current variance produces an increase in $\nu_{\text {out }}$ that is particularly significant if the input is subthreshold (Chance et al., 2002; Fellous et al., 2003). This is consistent with our simulations performed in both the FDR and the suprathreshold regimen in which increasing the input synchrony produced an increase in $\sigma_{\mathrm{I}}$, which translated to an increase in the rate (Fig. $7 A-D)$. An additional comparison of the results becomes difficult, because with depressing synapses, synchrony is not equivalent to a constant increase of $\sigma_{\mathrm{I}}$ but to a modulated variation that depends on the $\nu$ (Fig. 7A).

Although, in our simple LIF model, the non-monotonic re- sponse was obtained only under the FDR, it is possible that certain active membrane properties such as low-threshold, outward-rectifying currents (Reyes et al., 1994) may amplify the impact of synchrony in the suprathreshold regimen. In vitro experiments have shown that increasing the input fluctuations, at high mean drive, boosts the response rate, because large hyperpolarizing excursions allow the outward current to deactivate and hence enhance the neuron excitability (Reyes et al., 1996). This would imply that input synchrony combined with depression could modulate the response function in a non-monotonic manner even in the suprathreshold regimen.

Experimental (Chance et al., 2002) and theoretical studies (Kuhn et al., 2004; Moreno-Bote and Parga, 2005) have analyzed the effects of a change in the average conductance in the suprathreshold and subthreshold regimens, respectively. Using uncorrelated balanced inputs, they found that the increase of the mean conductance with $\nu$ leads to a decrease of the gain in the first case, or to a non-monotonic behavior of the response in the second. However, the non-monotonic response found in those works and the one found here are caused by different mechanisms, although in both cases the FDR is necessary. Whereas we increase the current fluctuations by introducing correlations that do not modify the total conductance substantially, the quoted studies increase $\sigma_{\mathrm{I}}$ by augmenting the afferent rates in a balanced manner, something that increases greatly the total conductance. Furthermore, as mentioned before, depression would prevent the linear increase of the conductance with $\nu$, so that an increasingly larger conductance could be achieved only by the recruitment of an increasingly larger number of presynaptic terminals rather than by a continuous increase in their firing rate.

We showed that the gain modulation of the non-monotonic response by the magnitude of the correlations is a robust effect. Systems showing selective neural response and gain modulation, which abound in visual areas and in parietal cortex (Salinas and Thier, 2000), are candidates in which this effect may occur. It is known that V1 cells receive, apart from visual information from the retina, a signal encoding the position of the eyes that modulates their gain (Trotter and Celebrini, 1999). It is plausible that this signal, which gates the flow of retinal information at the LGN (Lal and Friedlander, 1989), could control the degree of synchrony of geniculate cells [which can be very high (Alonso et al., 1996)]. Additionally, thalamocortical synapses show prominent STD (Stratford et al., 1996). Therefore, LGN cells exhibit synchrony and are affected by depression, the two required ingredients that, through the mechanism described here, could contribute to the observed gain effects.

\section{Appendix: Depression and the FDR}

A more accurate quantification of how much depression may constrain the target neuron to be in the subthreshold regimen can be performed using an LIF neuron model with synaptic conductances (Dayan and Abbot, 2001):

$$
C_{m} \frac{d V(t)}{d t}=-g_{L}\left(V(t)-E_{L}\right)-g_{e}(t)\left(V(t)-E_{\mathrm{rev}}\right) \quad \text { if } V<\theta .
$$

The excitatory synaptic conductance, $g_{e}(t)$, undergoes a transient change whenever a release takes place as described by the following:

$$
g_{e}(t)=G \exp \left(-\frac{t-t_{\mathrm{spk}}}{\tau_{s}}\right) H\left(t-t_{\mathrm{spk}}\right)
$$


where $t_{\mathrm{spk}}$ represents the spike time and $H(t)$ is the Heaviside function, which equals 1 for $t>0$ and 0 otherwise. Using this model, one can obtain an approximate expression for the average membrane potential (Kuhn et al., 2004) as follows:

$$
\langle V\rangle \approx \frac{g_{L} E_{L}+\left\langle g_{e}\right\rangle E_{\mathrm{rev}}}{g_{L}+\left\langle g_{e}\right\rangle} .
$$

Taking $E_{\mathrm{rev}}=0 \mathrm{mV}$ as the AMPA reversal potential and given that the average of the synaptic conductance reads $\left\langle g_{e}\right\rangle=N M G \tau_{s} \nu P_{t}$, one obtains the following:

$$
\langle V\rangle \approx \frac{g_{L} E_{L}}{g_{L}+N M G \tau_{s} \nu P_{t}} .
$$

The maximum $\langle V\rangle_{\max }$ is achieved when the release rate reaches its upper bound $\nu P_{t} \rightarrow \tau_{v}{ }^{-1}$. Substituting this expression into the condition $\langle V\rangle_{\max }\langle\theta$, which defines the FDR, we obtain the following:

$$
N<\frac{\left(E_{L}-\theta\right) g_{L} \tau_{v}}{\theta G M \tau_{s}} .
$$

Taking the values $E_{L}=-70 \mathrm{mV}, \theta=-50 \mathrm{mV}, \tau_{v}=500 \mathrm{~ms}, g_{L}=$ $1 / 60 \mu \mathrm{S}, \tau_{s}=3 \mathrm{~ms}$, and $M G=1.75 \mathrm{nS}$ (which produces a nondepressed EPSP of $1 \mathrm{mV}$ ), one obtains the boundary $N<630$. This rather large number supports the idea that depression may constrain a postsynaptic cell, receiving an input signal from a few hundred excitatory neurons firing at any rate, to work in the FDR.

\section{References}

Abbott LF, Varela JA, Sen K, Nelson SB (1997) Synaptic depression and cortical gain control. Science 275:179-180.

Alonso JM, Usrey WM, Reid RC (1996) Precisely correlated firing in cells of the lateral geniculate nucleus. Nature 383:815-819.

Baddeley RJ, Abbott LF, Booth M, Sengpiel F, Freeman T, Wakeman EA, Rolls ET (1997) Responses of neurons in primary and inferior temporal visual cortices to natural scenes. Proc R Soc Lond B Biol Sci 264:1775-1783.

Bair W, Koch C, Newsome W, Britten K (1994) Power spectrum analysis of bursting cells of area MT in the behaving monkey. J Neurosci 14:2870-2892.

Bernander O, Koch C, Usher M (1994) The effect of synchronized inputs at the single neuron level. Neural Comput 6:622-641.

Borg-Graham L, Monier C, Fregnac Y (1998) Visual input evokes transient and strong shunting inhibition in visual cortical neurons. Nature 393:369-373.

Braitenberg V, Schüz A (1991) Anatomy of the cortex: statistics and geometry. Berlin: Springer.

Buzsaki G, Draguhn A (2004) Neuronal oscillations in cortical networks. Science 304:1926-1929.

Calvin WH, Stevens C (1968) Synaptic noise and other sources of randomness in motoneuron interspike intervals. J Neurophysiol 31:574-587.

Chance FS, Nelson SB, Abbott LF (1998) Synaptic depression and the temporal response characteristics of V1 cells. J Neurosci 12:4785-4799.

Chance FS, Abbott L, Reyes AD (2002) Gain modulation from background synaptic input. Neuron 35:773-782.

Chung S, Li X, Nelson SB (2002) Short-term depression at thalamocortical synapses contributes to rapid adaptation of cortical sensory responses in vivo. Neuron 34:437-446.

Dan Y, Atick JJ, Reid RC (1996) Efficient coding of natural scenes in the lateral geniculate nucleus: experimental test of a computational theory. J Neurosci 16:3351-3362.

Davies C, Collingridge L (1990) Paired-pulsed depression of monosynaptic GABA-mediated inhibitory post-synaptic responses in rat hippocampus. J Physiol (Lond) 424:513-531.

Dayan P, Abbot LF (2001) Theoretical neuroscience: computational and mathematical modeling of neural systems, Ed 1. Cambridge, MA: MIT.

De Camilli P, Haucke V, Takei K, Mugnaini E (2001) The structure of synapses. In: Synapses, pp 89-133. Baltimore: Johns Hopkins University. de la Rocha J (2003) Computational consequences of short-term synaptic depression. PhD thesis, Universidad Autónoma de Madrid.

de la Rocha J, Nevado A, Parga N (2002) Information transmission by stochastic synapses with short-term depression: neural coding and optimization. Neurocomputing 44-46:85-90.

de la Rocha J, Moreno-Bote R, Parga N (2004) Correlations modulate the non-monotonic response of a neuron with short-term plasticity. Neurocomputing 58-60:313-319.

Dittman JS, Regehr WG (1998) Calcium dependence and recovery kinetics of presynaptic depression at the climbing fiber to Purkinje cell synapse. J Neurosci 18:6147-6162.

Dobrunz LE (2002) Release probability is regulated by the size of the readily releasable vesicle pool at excitatory synapses in hippocampus. Int J Dev Neurosci 730:1-12.

Dobrunz LE, Stevens CF (1997) Heterogeneity of release probability, facilitation, and depletion at central synapses. Neuron 18:995-1008.

Edwards F, Redman S, Walmsley B (1976) Statistical fluctuations in charge transfer at Ia synapses on spinal motoneurones. J Physiol (Lond) 259:665-688.

Fellous JM, Rudolph M, Destexhe A, Sejnowski TJ (2003) Synaptic background noise controls the input/output characteristics of single cells in an in vitro model of in vivo activity. Neuroscience 122:811-829.

Fuhrmann G, Segev I, Markram H, Tsodyks M (2002) Coding of temporal information by activity-dependent synapses. J Neurophysiol 87:140-148.

Fuhrmann G, Cowan A, Segev I, Tsodyks M, Stricker C (2004) Multiple mechanisms govern the dynamics of depression at neocortical synapses of young rats. J Physiol (Lond) 557:415-438.

Galarreta M, Hestrin S (1998) Frequency-dependent synaptic depression and the balance of excitation and inhibition in the neocortex. Nat Neurosci 1:587-594.

Gerstein G, Mandelbrot B (1964) Random walk models for the spike activity of a single neuron. Biophys J 71:41-68.

Gil Z, Connors BW, Amitai Y (1999) Efficacy of thalamocortical and intracortical synaptic connections: quanta, innervation, and reliability. Neuron 23:385-397.

Goldberg J, Brown P (1969) Response of binaural neurons of dog superior olivary complex to dichotic tonal stimuli: some physiological mechanisms of sound localization. J Neurophysiol 32:613-636.

Goldman MS, Maldonado P, Abbott LF (2002) Redundancy reduction and sustained firing with stochastic depressing synapses. J Neurosci 22:584-591.

Grande LA, Kinney GA, Miracle GL, Spain WJ (2004) Dynamic influences on coincidence detection in neocortical pyramidal neurons. J Neurosci 24:1839-1851

Gupta A, Wang Y, Markram H (2000) Organizing principles for a diversity of GABAergic interneurons and synapses in the neocortex. Science 287:273-278.

Hanse E, Gustafsson B (2001) Vesicle release probability and pre-primed pool at glutamatergic synapses in area cal of the rat neonatal hippocampus. J Physiol (Lond) 531:481-493.

Held H (1893) Die centrale gehorleitung. Arch Anat Physiol 17:201-248.

Hessler N, Shirke A, Malinow R (1993) The probability of transmitter release at a mammalian central synapse. Nature 366:569-572.

Hsu S, Augustine G, Jackson M (1996) Adaptation of $\mathrm{Ca}^{2+}$-triggered exocytosis in presynaptic terminals. Neuron 17:501-512.

Katz B, Miledi R (1968) The role of calcium in neuromuscular facilitation. J Physiol (Lond) 195:481-492.

Krahe R, Gabbiani F (2004) Burst firing in sensory systems. Nat Rev Neurosci 5:13-23.

Kuhn A, Aertsen A, Rotter S (2003) Higher-order statistics of input ensembles and the response of simple model neurons. Neural Comput 15:67-101.

Kuhn A, Aertsen A, Rotter S (2004) Neuronal integration of synaptic input in the fluctuation-driven regime. J Neurosci 24:2345-2356.

Kyriazi H, Simons D (1993) Thalamocortical response transformations in simulated whisker barrels. J Neurosci 13:1601-1615.

Lal R, Friedlander M (1989) Gating of retinal transmission by afferent eye position and movement signals. Science 243:93-96.

Lisman JE (1997) Bursts as a unit of neural information: making unreliable synapses reliable. Trends Neurosci 20:38-43.

Maass W, Zador AM (1999) Dynamic stochastic synapses as computational units. Neural Comput 11:903-917. 
Magleby KL (1987) Short-term changes in synaptic efficacy. In: Synaptic function (Edelman G, Gall W, Cowan W, eds), pp 21-56. New York: Wiley.

Markram H, Tsodyks M (1996) Redistribution of synaptic efficacy between neocortical pyramidal neurons. Nature 382:807-810.

Markram H, Lübke J, Protscher M, Roth A, Sakmann B (1997) Physiology and anatomy of synaptic connections between thick tufted pyramidal neurones in the developing rat neocortex. J Physiol (Lond) 500:409-440.

Markram H, Wang Y, Tsodyks M (1998) Differential signaling via the same axon of neocortical pyramidal neurons. Proc Natl Acad Sci USA 95:977-979.

Matveev V, Wang X-J (2000a) Implications of all-or-none synaptic transmission and short-term depression beyond vesicle depletion: a computational study. J Neurosci 20:1575-1588.

Matveev V, Wang X-J (2000b) Differential short-term synaptic plasticity and transmission of complex spike trains: to depress or to facilitate? Cereb Cortex 10:1143-1153.

Moreno R, de la Rocha J, Parga N (2002a) Response of a leaky integrate and fire (LIF) neuron when stimulated with synapses showing short-term depression. Soc Neurosci Abstr 28:752.6.

Moreno R, de la Rocha J, Renart A, Parga N (2002b) Response of spiking neurons to correlated inputs. Phys Rev Lett 89:288101.

Moreno-Bote R, Parga N (2005) Membrane potential and response properties of populations of cortical neurons in the high conductance state. Phys Rev Lett 94:088103.

Murthy VN, Fetz EE (1994) Effects of input synchrony on the firing rate of a three-conductance cortical neuron model. Neural Comput 6:1111-1126.

Murthy V, Schikorski T, Stevens CF, Yongling Z (2001) Inactivity produces increases in neurotransmitter release and synapse size. Neuron 32:673-682.

Pedroarena CM, Schwarz C (2003) Efficacy and short-term plasticity at GABAergic synapses between Purkinje and cerebellar nuclei neurons. J Neurophysiol 89:704-715.

Phillips D, Irvine DRF (1981) Responses of single units in physiologically defined primary auditory cortex (AI) of the cat: frequency tuning and response to intensity. J Neurophysiol 45:48-58.

Reyes A, Rubel E, Spain W (1994) Membrane properties underlying the firing of neurons in the avian cochlear nucleus. J Neurosci 14:5352-5364.

Reyes A, Rubel E, Spain W (1996) In vitro analysis of optimal stimuli for phase-locking and time-delayed modulation of firing in avian nucleus laminaris neurons. J Neurosci 16:993-1007.

Ricciardi LM (1977) Diffusion processes and related topics in biology. Berlin: Springer.

Rosenmund C, Clements J, Westbrook G (1993) Nonuniform probability of glutamate release at a hippocampal synapse. Science 262:754-757.

Salinas E, Abbott LF (1996) A model of multiplicative neural responses in parietal cortex. Proc Natl Acad Sci USA 93:11956-11961.

Salinas E, Sejnowski TJ (2000) Impact of correlated synaptic input on output firing rate and variability in simple neuronal models. J Neurosci 20:6193-6209.

Salinas E, Thier P (2000) Gain modulation: a major computational principle of the central nervous system. Neuron 27:15-21.

Scanziani M, Salin PA, Vogt KE, Malenka RC, Nicoll RA (1997) Usedependent increases in glutamate concentration activate presynaptic metabotropic glutamate receptors. Nature 385:630-634.

Schneggenburger R, Sakaba T, Neher E (2002) Vesicle pools and short-term synaptic depression: lessons from a large synapse. Trends Neurosci 25:206-212.

Shadlen MN, Newsome WT (1994) Noise, neural codes and cortical organization. Curr Opin Neurobiol 4:569-579.
Shadlen MN, Newsome WT (1998) The variable discharge of cortical neurons: implications for connectivity, computation, and information coding. J Neurosci 18:3870-3896.

Shelley M, McLaughlin D, Shapley R, Wielaard J (2002) States of high conductance in a large-scale model of the visual cortex. J Comput Neurosci 13:93-109.

Sherman SM (2001) Tonic and burst firing: dual modes of thalamocortical relay. Trends Neurosci 24:122-126.

Silver RA, Lubke J, Sakmann B, Feldmeyer D (2003) High-probability uniquantal transmission at excitatory synapses in barrel cortex. Science 302:1981-1984.

Softky W, Koch C (1993) The highly irregular firing of cortical cells is inconsistent with temporal integration of random EPSPs. J Neurosci 13:334-350.

Stevens C, Wesseling J (1998) Activity-dependent modulation of the rate at which synaptic vesicles become available to undergo exocytosis. Neuron 20:1243-1253.

Stevens CF, Wang Y (1994) Changes in reliability of synaptic function as a mechanism for plasticity. Nature 371:704-707.

Stratford K, Tarczy-Hornoch K, Martin K, Bannister N, Jack J (1996) Excitatory synaptic inputs to spiny stellate cells in cat visual cortex. Nature 382:258-261.

Sutter ML, Schreiner CE (1995) Topography of intensity tuning in cat primary auditory cortex: single-neuron versus multiple-neuron recordings. J Neurophysiol 73:190-204.

Thomson A (1997) Activity-dependent properties of synaptic transmission at two classes of connections made by rat neocortical pyramidal axons in vitro. J Physiol (Lond) 502:131-147.

Trotter Y, Celebrini S (1999) Gaze direction controls response gain in primary visual-cortex neurons. Nature 398:239-242.

Troyer T, Krukowski A, Priebe N, Miller K (1998) Contrast-invariant orientation tuning in cat visual cortex: thalamocortical input tuning and correlation-based intracortical connectivity. J Neurosci 18:5908-5927.

Tsodyks MV, Markram H (1997) The neural code between neocortical pyramidal neurons depends on neurotransmitter release probability. Proc Natl Acad Sci USA 94:719-723.

van Vreeswijk C, Sompolinsky H (1996) Chaos in neural networks with balanced excitatory and inhibitory activity. Science 274:1724-1726.

Varela J, Sen K, Gibson J, Fost J, Abbott L, Nelson S (1997) A quantitative description of short-term plasticity at excitatory synapses in layer $2 / 3$ of rat primary visual cortex. J Neurosci 17:7926-7940.

Varela JA, Song S, Turrigiano GG, Nelson SB (1999) Differential depression at excitatory and inhibitory synapses in visual cortex. J Neurosci 19:4293-4304.

Vere-Jones D (1966) Simple stochastic models for the release of quanta of transmitter from a nerve terminal. Aust J Stat 8:53-63.

Walmsley B (1991) Central synaptic transmission: studies at the connection between primary afferent fibres and dorsal spinocerebellar tract (DSCT) neurones in Clarke's column of the spinal cord. Prog Neurobiol 36:391-423.

Walmsley B, Alvarez FJ, Fyffe REW (1998) Diversity of structure and function at mammalian central synapses. Trends Neurosci 21:81-88.

Wang L-Y, Kaczmarek LK (1998) High-frequency firing helps replenish the readily releasable pool of synaptic vesicles. Nature 394:384-388.

Wang X-J (1999) Fast burst firing and short-term synaptic plasticity: a model of neocortical chattering neurons. Neuroscience 89:347-362.

Zador A (1998) The impact of synaptic unreliability on the information transmitted by spiking neurons. J Neurophysiol 79:1219-1229.

Zucker RS, Regehr WG (2002) Short-term synaptic plasticity. Annu Rev Physiol 64:355-405. 